\title{
Quasi-permutation modules over finite groups
}

\author{
By Shizuo ENDo and Takehiko MIYATA
}

(Received March 27, 1972)

Let $\Pi$ be a finite group. A finitely generated $Z$-free $\Pi$-module is briefly called a $\Pi$-module. A $\Pi$-module is called a permutation $\Pi$-module if it is expressible as a direct sum of some $\left\{Z \Pi / \Pi_{i}\right\}$ where each $\Pi_{i}$ is a subgroup of $\Pi$. Further a $\Pi$-module $M$ is called a quasi-permutation $\Pi$-module if there exists an exact sequence: $0 \rightarrow M \rightarrow S \rightarrow S^{\prime} \rightarrow 0$ where $S$ and $S^{\prime}$ are permutation $\Pi$-modules.

In [2] we have studied the properties of quasi-permutation modules in relation with a problem in invariant theory. In this paper we will give some basic results on quasi-permutation modules as a continuation to [2].

First we will consider projective quasi-permutation $\Pi$-modules.

Let $R$ be a Dedekind domain and $K$ be the quotient field of $R$. Let $\Sigma$ be a separable $K$-algebra and $\Lambda$ be an $R$-order in $\Sigma$. Denote by $\boldsymbol{P}(\Lambda)$ the set of all isomorphism types of finitely generated projective (left) $\Lambda$-modules and put $\boldsymbol{P}_{0}(\Lambda)=\{[P] \in \boldsymbol{P}(\Lambda) \mid P$ is locally free $\}$. Let $P_{0}(\Lambda)$ be the Grothendieck group of $\boldsymbol{P}_{0}(\Lambda)$. We define an epimorphism $\mu_{\Lambda}: P_{0}(\Lambda) \rightarrow Z$ by $\mu_{\Lambda}\left(\left[P_{1}\right]-\left[P_{2}\right]\right)=$ $\operatorname{rank}_{\Sigma}^{*} K P_{1}-\operatorname{rank}_{\Sigma} K P_{2}$. Now we put $C(\Lambda)=\operatorname{Ker} \mu_{\Lambda}$ and call this the (reduced) projective class group of $\Lambda$ (cf. [5], [11]). Especially, if $\Lambda$ is commutative, then $C(\Lambda)$ is isomorphic to the Picard group of $\Lambda$. Further let $\Omega$ be a maximal $R$-order in $\Sigma$ which contains $\Lambda$. We define a homomorphism $: \nu_{\Omega / \Lambda}: C(\Lambda) \rightarrow C(\Omega)$ by $\nu_{\Omega / \Lambda}\left(\left[P_{1}\right]-\left[P_{2}\right]\right)=\left[\Omega \bigotimes_{\Lambda} P_{1}\right]-\left[\Omega \otimes_{\Lambda} P_{2}\right]$. Then it is known that $\nu_{\Omega / \Lambda}$ is an epimorphism but not always a monomorphism. Hence putting $\tilde{C}(\Lambda)=\operatorname{Ker} \nu_{\Omega_{1} / \Lambda}$, we have an exact sequence:

$$
0 \longrightarrow \tilde{C}(\Lambda) \longrightarrow C(\Lambda) \longrightarrow C(\Omega) \longrightarrow 0 \text {. }
$$

Especially let $\Lambda=Z \Pi$ and let $\Omega_{\Pi}$ be a maximal order in $Q \Pi$ which contains $Z \Pi$. Then, by the Swan's theorem $([\mathbf{1 1}])$, we have $\boldsymbol{P}_{0}(Z \Pi)=\boldsymbol{P}(Z \Pi)$ and $\tilde{C}(Z \Pi)=\{[\mathfrak{a}]-[Z \Pi] \in C(Z \Pi) \mid \mathfrak{a}$ is a projective (left) ideal of $Z \Pi$ such that $\Omega_{\Pi} \mathfrak{a} \oplus \Omega_{\Pi} \cong \Omega_{\Pi} \oplus \Omega_{\Pi}$ as $\Omega_{\Pi}$-modules\}. It is noted that $\tilde{C}(Z \Pi)$ does not depend on the choice of $\Omega_{\Pi}$ (cf. [3]). On the other hand, we put $C^{q}(Z \Pi)=$ $\{[\mathfrak{a}]-[Z \Pi] \in C(Z \Pi) \mid \mathfrak{a}$ is a quasi-permutation projective (left) ideal of $Z \Pi\}$. Then it is easily seen that $C^{q}(Z \Pi)$ is also a subgroup of $C(Z \Pi)$.

Let $\Pi$ be a cyclic group of order $n$ and $\sigma$ be a generator of $\Pi$. We 
denote by $\Phi_{m}(T)$ the $m$-th cyclotomic polynomial and by $\zeta_{m}$ a primitive $m$-th root of unity. Let $M$ be a $\Pi$-module and put $M^{\phi_{m}}=\left\{u \in M \mid \Phi_{m}(\sigma) u=0\right\}$ for any $m \mid n$. Then $Z \Pi /\left(\Phi_{m}(\sigma)\right) \cong Z\left[\zeta_{m}\right]$ and $M^{\Phi_{m}}$ can be regarded as a $Z\left[\zeta_{m}\right]$ module for any $m \mid n$. In [2], (1.11) we have proved that a projective $\Pi$-module $P$ is a quasi-permutation $\Pi$-module if and only if, for any $m \mid n, P^{\Phi_{m}}$ is $Z\left[\zeta_{m}\right]$ free. This is clearly equivalent to the assertion that $\widetilde{C}(Z \Pi)=C^{q}(Z \Pi)$.

In this paper we will first give, as a generalization of this result,

[I] For any finite abelian group $\Pi, \widetilde{C}(Z \Pi)=C^{q}(Z \Pi)$.

It seems natural to ask whether $C^{q}(Z \Pi)$ coincides with $\tilde{C}(Z \Pi)$ for any finite group $\Pi$ or not. In fact, we will prove

[II] Let $I I$ be one of the following groups:

(i) p-groups where $p$ is an odd prime;

(ii) dihedral groups, $D_{p} l$, where $p$ is a prime and $l$ is a positive integer;

(iii) the quaternion group $H_{2}$, the alternating group $A_{4}$ and the symmetric group $S_{4}$.

Then $\tilde{C}(Z \Pi)=C^{q}(Z \Pi)$.

To prove [II] we use the Jacobinski-Roiter's results in [3] and [6]. Furthermore using them, we can show the following refinement of [I].

[III] Let $\Pi$ be a finite abelian group and let a be a projective (left) ideal of $Z \Pi$. Then a is a quasi-permutation $\Pi$-module if and only if $\mathfrak{a} \oplus \sum_{\Pi^{\prime} \in \mathscr{S}} \oplus Z \Pi / \Pi^{\prime}$ $\cong Z \Pi \bigoplus_{\Pi^{\prime} \in \subseteq^{\prime}} \oplus Z \Pi / \Pi^{\prime}$, where $\mathfrak{S}$ denotes the set of all subgroups, $\Pi^{\prime}(\neq 1)$, of $\Pi$ such that $\Pi / \Pi^{\prime}$ is cyclic.

It is much more difficult to examine the properties of non projective quasipermutation modules. Here we will consider only the case where $\Pi$ is a cyclic group.

[IV] Let $\Pi$ be a cyclic p-group of order $p^{l}$.

(i) A II-module $M$ is a quasi-permutation module if and only if, for any $0 \leqq m \leqq l, M^{\Phi} p^{m}$ is a free $Z\left[\zeta_{p m}\right]$-module.

(ii) If $M$ is a quasi-permutation $\Pi$-module, then the dual module $M^{*}=$ $\operatorname{Hom}_{Z}(M, Z)$ of $M$ is also a quasi-permutation $\Pi$-module.

(iii) Let $0 \rightarrow M^{\prime} \rightarrow M \rightarrow M^{\prime \prime} \rightarrow 0$ be an exact sequence of II-modules. If two of $M^{\prime}, M$ and $M^{\prime \prime}$ are quasi-permutation $\Pi$-modules, then the rest of them is a quasi-permutation $\Pi$-module.

Let $\Pi$ be a finite group and $K / k$ be a Galois extension with group $\cong$. Let $M$ be a $\Pi$-module with a $Z$-free basis $\left\{u_{1}, u_{2}, \cdots, u_{n}\right\}$. Denote by $K\left(X_{1}, X_{2}\right.$, $\left.\cdots, X_{n}\right)$ the rational function field with $n$-variables $X_{1}, X_{2}, \cdots, X_{n}$ over $K$ and define the action of $I I$ on $K\left(X_{1}, X_{2}, \cdots, X_{n}\right)$, as an extension of the action of $\Pi$ on $K$, by putting

$$
\sigma\left(X_{i}\right)=\prod_{j=1}^{n} X_{j}^{m_{i j}} \quad \text { when } \quad \sigma \cdot u_{i}=\sum_{j=1}^{n} m_{i j} u_{j}, \quad m_{i j} \in Z
$$


for any $\sigma \in \Pi$ and $1 \leqq i \leqq n$. We denote $K\left(X_{1}, X_{2}, \cdots, X_{n}\right)$ with this action of $\Pi$ by $K(M)$.

[V] Let $\Pi$ be a cyclic p-group and $K / k$ be a Galois extension with group $\cong I I$. In case of $p \neq 2$, assume that $k$ is infinite. If $M$ is a quasi-permutation $\Pi$-module, then $K(M)^{\prime \prime} / k$ is rational.

$\S 1$. We give, as a slight generalization of [2], (1.1),

Proposition 1.1. Let $K / k$ be a Galois extension with group $\Pi$ and $K\left(X_{1}, X_{2}\right.$, $\left.\cdots, X_{n}\right)$ be the rational function field with $n$ variables $X_{1}, X_{2}, \cdots, X_{n}$ over $K$. Further suppose that $\Pi$ acts on $K\left(X_{1}, X_{2}, \cdots, X_{n}\right)$ as follows:

$$
\sigma\left(X_{i}\right)=\sum_{i=1}^{n} \alpha_{i j}(\sigma) X_{j}+\beta_{i}(\sigma), \quad \alpha_{i j}(\sigma), \quad \beta_{i}(\sigma) \in K .
$$

Then $K\left(X_{1}, X_{2}, \cdots, X_{n}\right)^{I I}$ is rational over $k$.

PROOF. We denote by $A f f(n, K)$ the affine transformation group of the $n$-dimensional affine space over $K$. Then we have an exact sequence of $\Pi$ groups :

$$
1 \longrightarrow K^{(n)} \longrightarrow A f f(n, K) \longrightarrow G L(n, K) \longrightarrow 1 \text {. }
$$

From this we get an exact sequence:

$$
H^{1}(\Pi, K)^{(n)} \longrightarrow H^{1}(\Pi, A f f(n, K)) \longrightarrow H^{1}(\Pi, G L(n, K)) .
$$

By the Hilbert's theorem $90 H^{1}(\Pi, K)^{(n)}=H^{1}(I, G L(n, K))=1$, and so $H^{1}(\Pi$, $A f f(n, K))=1$. The proposition is clearly a restatement of the fact that $H^{1}(\Pi, A f f(n, K))=1$ (cf. [2]).

Let $E, F$ be extensions of a field $k$. We define a relation $E_{-{ }_{(r)_{k}}} F$ if there exist variables $X_{1}, X_{2}, \cdots, X_{s}$ and $Y_{1}, Y_{2}, \cdots, Y_{t}$ such that $E\left(X_{1}, X_{2}, \cdots, X_{s}\right)$ is $k$-isomorphic to $F\left(Y_{1}, Y_{2}, \cdots, Y_{t}\right)$. An extension $E / k$ is said to be quasirational if $E \frac{-(r)_{k}}{} k$.

Let $\Pi$ be a finite group and denote by $C_{Z \Pi}$ the class of all $\Pi$-modules. Let $M, N \in C_{Z \Pi \text {. }}$. We define an equivalence relation $M \frac{{ }_{(r)}}{}-N$ if, for any Galois extension $K / k$ with group $\cong \Pi, K(M)^{\Pi} \frac{-(r)_{k}}{(n)} K(N)^{\Pi}$. If $M_{1} \frac{(r)}{{ }^{\prime}} N_{1}$ and $M_{2}{ }_{(r)} N_{2}$, then $M_{1} \oplus M_{2}-(r)-N_{1} \oplus N_{2}$. Let $T(\Pi)$ be the set of all equivalence classes in $\boldsymbol{C}_{Z \Pi \text {. }}$. We define an addition in $T(\Pi)$ by $[M]+[N]=[M \oplus N]$. Then this makes $T(\Pi)$ an abelian semigroup.

Further let $M, N \in C_{Z \Pi}$. We write $M \overline{\overline{(r)}} N$ if, for any Galois extension $K / k$ with group $\cong \Pi, K(M)^{\Pi}$ is $k$-isomorphic to $K(N)^{\Pi}$.

The following fundamental theorem is essentially due to R. G. Swan (cf. [13], [14]. Also see [2], (1.6).)

THEOREM 1.2. Let $I I$ be a finite group and let $M$ be a $\Pi$-module. Then the following conditions are equivalent:

(1) $M$ is a quasi-permutation $\Pi$-module. 
(2) For a fixed Galois extension $K / k$ with group $\cong \Pi, K(M)^{I} / k$ is quasirational.

(3) For any Galois extension $K / k$ with group $\cong \Pi, K(M)^{\Pi} / k$ is quasi-rational, i.e., $M-\overline{(r)} 0$.

COROLlary 1.3. Let $\Pi$ be a finite group. Let

$$
0 \longrightarrow M \longrightarrow N \longrightarrow S \longrightarrow 0
$$

be an exact sequence where $M, N$ are $\Pi$-modules and $S$ is a permutation $\Pi$ module. Then $N=M \oplus S$ and $N-(r)-M$. Especially $N$ is a quasi-permutation $I I$-module if and only if $M$ is a quasi-permutation II-module.

Proposition 1.4. Let $\Pi$ be a finite group. Let $M$ be a $\Pi$-module and $L$ be a quasi-permutation $I I$-module. If $M \oplus L$ is a quasi-permutation $\Pi$-module, then $M$ is also a quasi-permutation $\Pi$-module.

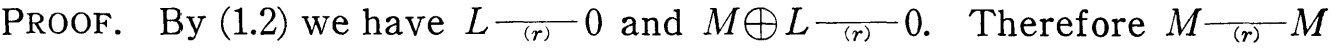
$\oplus L-(r) 0$. Again by (1.2) we can conclude that $M$ is a quasi-permutation $\Pi I$-module.

LeMMA 1.5. Let $I I$ be a finite group. Let $M$ be a $\Pi$-module and let $\Pi_{1}^{\prime}, \cdots, \Pi_{s}^{\prime}$ be subgroups of $\Pi$. Then the following conditions are equivalent:

(1) $H^{1}\left(\Pi_{i}^{\prime}, M\right)=0$ for any $1 \leqq i \leqq s$.

(2) Every exact sequence $0 \rightarrow M \rightarrow N \rightarrow \sum_{i=1}^{s} \oplus\left(Z \Pi / \Pi_{i}^{\prime}\right)^{\left(t_{i}\right)} \rightarrow 0$, where $t_{1}, \cdots, t_{s}$ are non-negative integers, splits.

Proof. For any $\Pi$-module $L$ and any subgroup $\Pi^{\prime}$ of $\Pi$ there exists a natural isomorphism $H^{1}\left(\Pi^{\prime}, L\right) \cong \operatorname{Ext}_{Z n}^{1}\left(Z \Pi / \Pi^{\prime}, L\right)$. From this the lemma follows immediately.

Proposition 1.6. Let $\Pi$ be a finite group. For any $\Pi$-module $M$ the following conditions are equivalent:

(1) $M$ is a quasi-permutation $\Pi$-module and $H^{1}\left(\Pi^{\prime}, M\right)=0$ for any subgroup $\Pi^{\prime}$ of $\Pi$.

(2) There exist permutation $\Pi$-modules $S, S^{\prime}$ such that $M \oplus S^{\prime} \cong S$. Especially a projective $\Pi$-module $P$ is a quasi-permutation $\Pi$-module if and only if there exist permutation $\Pi$-modules $S$, $S^{\prime}$ such that $P \oplus S^{\prime} \cong S$.

ProOF. Assume (1). Then there is an exact sequence $0 \rightarrow M \rightarrow S \rightarrow S^{\prime} \rightarrow 0$ where $S$ and $S^{\prime}$ are permutation $\Pi$-modules. By (1.5) this sequence splits, and hence $M \oplus S^{\prime} \cong S$. This proves $(1) \Rightarrow(2)$. Conversely assume (2). Then it is clear by the definition that $M$ is a quasi-permutation $\Pi$-module. Further we see that $H^{1}\left(\Pi^{\prime}, S\right)=H^{1}\left(\Pi^{\prime}, S^{\prime}\right)=0$ for any subgroup $\Pi^{\prime}$ of $\Pi$. Therefore $H^{1}\left(\Pi^{\prime}, M\right)=0$ for any subgroup $\Pi^{\prime}$ of $\Pi$. Thus $(2) \Rightarrow(1)$. The second part of the proposition follows from the first part.

It should be noted that the second part of (1.6) can be directly proved by dualizing the exact sequence $0 \rightarrow P \rightarrow S \rightarrow S^{\prime} \rightarrow 0$ where $S, S^{\prime}$ are permutation $\Pi$-modules. 
Proposition 1.7. For any finite group $\Pi, C^{q}(Z \Pi)$ is a subgroup of $C(Z \Pi)$.

Proof. Let $a$ be a quasi-permutation, projective ideal of $Z \Pi$. Then there exists a projective ideal $\mathfrak{b}$ of $Z \Pi$ such that $\mathfrak{a} \oplus \mathfrak{b} \cong Z \Pi \oplus Z \Pi$. By (1.6) (or by (1.4)) $\mathfrak{b}$ is a quasi-permutation $\Pi$-module and we have $-([\mathfrak{a}]-[Z \Pi])=$ $[\mathrm{b}]-[Z \Pi]$.

$\S 2$. Let $\Pi$ be a cyclic group of order $n$ and $\sigma$ be a generator of $\Pi$. Let $Z[T]$ be the polynomial ring with a variable $T$ over $Z$ and $\Phi, \Psi$ be monic polynomials of $Z[T]$ such that $\Phi \cdot \Psi=T^{n}-1$. If $M$ is a $\Pi$-module, there are three ways to construct a $Z \Pi /(\Phi(\sigma))$-module from $M$, i. e., putting $\Psi M=$ $\Psi(\sigma) M, M_{\Phi}=M / \Phi(\sigma) M$ and $M^{\Phi}=\{u \in M \mid \Phi(\sigma) u=0\} \cong \operatorname{Hom}_{Z \Pi}(Z \Pi /(\Phi(\sigma)), M)$, $\Psi M, M_{\Phi}$ and $M^{\Phi}$ can be regarded as $Z \Pi /(\Phi(\sigma))$-modules. Then $\Psi M \subseteq M^{\Phi}$. We define an epimorphism $\theta_{M}: M_{\oplus} \rightarrow \Psi M$ by $\theta_{M}(\bar{u})=\Psi(\sigma) u$. Especially, if we take the $m$-th cyclotomic polynomial $\Phi_{m}(T)(m \mid n)$ as $\Phi(T)$, we have $Z \Pi /\left(\Phi_{m}(\sigma)\right) \cong Z\left[\zeta_{m}\right]$ where $\zeta_{m}$ denotes the primitive $m$-th root of unity. We can easily prove the following two lemmas (cf. [13]).

LEMMA 2.1. Let $\Pi$ be a cyclic group of order $n$ and $\sigma$ be a generator of II. For any positive integers $m, l$ dividing $n$, we have

$$
\left(Z \Pi /\left[\sigma^{l}\right]\right)^{\Phi_{m}} \cong \begin{cases}Z\left[\zeta_{m}\right] & \text { when } m \mid l \\ 0 & \text { when } m+l .\end{cases}
$$

If $S$ is a permutation $\Pi$-module, then $S^{\Phi_{m}}$ is a free $Z\left[\zeta_{m}\right]$-module.

LEMMA 2.2. Let $\Pi$ be a cyclic group of order $n$ and $\Phi(T), \Psi(T)$ be monic polynomials such that $\Phi(T) \Psi(T)=T^{n}-1$. Let $P$ be a projective $\Pi$-module. Then $\Psi P=P^{\Phi}$ and $\theta_{P}: P_{\Phi} \rightarrow \Psi P$ is an isomorphism.

The following proposition has been proved essentially in [2], (1.11).

Proposition 2.3. Let II be a finite abelian group. Then the maximal order $\Omega_{\Pi}$ of $Q \Pi$ which contains $Z \Pi$ is a quasi-permutation $\Pi$-module.

Proof. We can express $\Omega_{\Pi}$ as a direct sum of $Z[\zeta]$ 's where each $\zeta$ is the root of unity. Therefore it suffices to prove that each $Z[\zeta]$ is a quasipermutation $\Pi$-module. Let us denote by $\Pi^{\prime}$ the kernel of the natural projection of $\Pi$ on $[\zeta]$. Then $\Pi / \Pi^{\prime}$ is cyclic and $Z[\zeta]$ can be regarded as a $\Pi / \Pi^{\prime}$-module. Hence we may suppose that $\Pi$ is a cyclic group of order $n$ with a generator $\sigma$ and $Z[\zeta] \cong Z \Pi /\left[\Phi_{n}(\sigma)\right]$. Then, using the Möbius' inversion formula, we obtain the following exact sequences of $\Pi$-modules :

$$
\begin{aligned}
& 0 \longrightarrow M_{1} \longrightarrow Z \Pi \longrightarrow Z \Pi / \Pi_{0} \longrightarrow 0 \\
& 0 \longrightarrow M_{1} \longrightarrow M_{2} \longrightarrow Z \Pi / \Pi_{1} \longrightarrow 0 \\
& 0 \longrightarrow M_{3} \longrightarrow M_{2} \longrightarrow Z \Pi / \Pi_{2} \longrightarrow 0
\end{aligned}
$$




$$
\begin{aligned}
& 0 \longrightarrow M_{t-1} \longrightarrow M_{t} \longrightarrow Z \Pi / \Pi_{t-1} \longrightarrow 0 \\
& 0 \longrightarrow Z \Pi /\left(\Phi_{n}(\sigma)\right) \longrightarrow M_{t} \longrightarrow Z \Pi / \Pi_{t} \longrightarrow 0,
\end{aligned}
$$

where $\Pi_{0}, \cdots, \Pi_{t}$ are subgroups of $\Pi$. Applying (1.3) to these exact sequences repeatedly, we see that $Z \Pi /\left(\Phi_{n}(\sigma)\right)$ is a quasi-permutation $\Pi$-module.

LEMMA 2.4. Let $\Pi$ be a finite group and a be a projective (left) ideal of $Z \Pi$. Let $\Lambda$ be an order in $Q \Pi$ which contains $Z \Pi$. Then $\mathfrak{a} \oplus \Lambda \cong Z \Pi \oplus \Lambda \mathfrak{a}$ as $\Pi$-modules.

PROOF. Let $n$ be the order of $\Pi$. Then we know $n \Lambda \subseteq Z \Pi$ and there exists an ideal $\mathfrak{a}_{0}$ of $Z \Pi$ such that $\mathfrak{a}_{0} \cong \mathfrak{a}$ and $\left(\operatorname{Ann}_{Z} \Lambda / \mathfrak{a}_{0}, n\right)=1([\mathbf{1 1}])$. Hence we may assume that $\left(\operatorname{Ann}_{z} \Lambda / \mathfrak{a}, n\right)=1$. Now we can make the commutative diagram with exact rows:

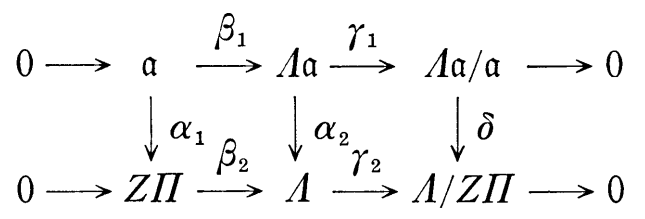

where $\alpha_{1}, \alpha_{2}, \beta_{1}, \beta_{2}$ are natural injections. Let $p$ be a rational prime. If $p+n$, then $Z_{p} \Pi$ is a maximal $Z_{p}$-order in $Q \Pi$, therefore both $\left(\beta_{1}\right)_{p}$ and $\left(\beta_{2}\right)_{p}$ are isomorphisms. Hence $\delta_{p}(=0)$ is an isomorphism. On the other hand, if $p \mid n$, then $p+\operatorname{Ann}_{Z} Z \Pi / a$ so that both $\left(\alpha_{1}\right)_{p}$ and $\left(\alpha_{2}\right)_{p}$ are isomorphisms, hence $\delta_{p}$ is also an isomorphism. Accordingly, for any $p, \delta_{p}$ is an isomorphism. Thus $\delta$ must be an isomorphism. We identify $\Lambda \mathfrak{a} / \mathfrak{a}$ with $\Lambda / Z \Pi$ through $\delta$ and denote it by $A$. Forming the pullback of $\gamma_{1}$ and $\gamma_{2}$, we get the following commutative diagram with exact rows and columns:

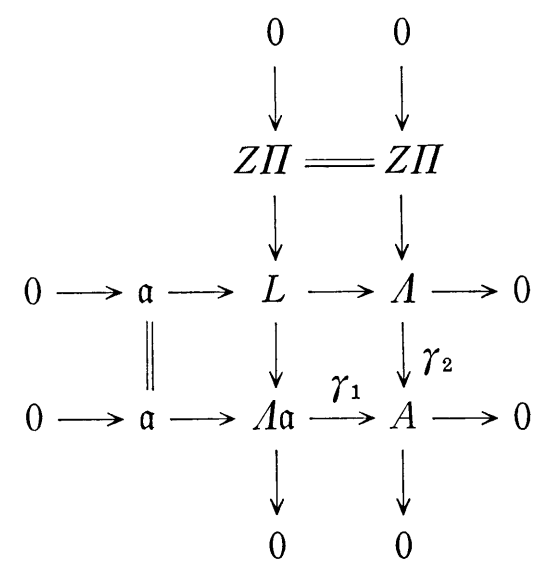

Since $a, Z \Pi$ are $\Pi$-projective and $L, \Lambda \mathfrak{a}, \Lambda$ are torsion-free, the second row and the second column are $\Pi$-split. Therefore $Z \Pi \oplus \Lambda \mathfrak{a} \cong L \cong \mathfrak{a} \oplus \Lambda$, which completes the proof. 


\section{Now we prove}

THEOREM 2.5. Let $\Pi$ be a finite abelian group. Then

$$
\tilde{C}(Z \Pi)=C^{q}(Z \Pi) \text {. }
$$

Proof. Let $\mathfrak{a}$ be a projective ideal of $Z \Pi$ and let $\Omega_{\Pi}$ be the maximal order in $Q \Pi$ which contains $Z \Pi$. By the definitions of $\tilde{C}(Z \Pi)$ and $C^{q}(Z \Pi)$, it suffices to prove that $a$ is a quasi-permutation $\Pi$-module if and only if $\Omega_{\Pi} \mathfrak{a} \cong \Omega_{\Pi}$ as $\Omega_{\Pi}$-modules.

Suppose that $\mathfrak{a}$ is a quasi-permutation $\Pi$-module. Then there exist permutation $\Pi$-modules $S, S^{\prime}$ such that $\mathfrak{a} \oplus S^{\prime} \cong S$ by (1.6). Since $\Pi$ is abelian, $\Omega_{\Pi}^{-}$is expressible as the direct sum of $Z\left[\zeta_{m}\right]^{\prime}$ s where each $\zeta_{m}$ is a primitive $m$-th root of unity. To show $\Omega_{n} \mathfrak{a} \cong \Omega_{n}$, it suffices to show $Z\left[\zeta_{m}\right] \mathfrak{a} \cong Z\left[\zeta_{m}\right]$ for every component $Z\left[\zeta_{m}\right]$ of $\Omega_{\Pi}$. We denote by $\Pi^{\prime}$ the kernel of the natural projection of $\Pi$ on $\left[\zeta_{m}\right]$. Then $\Pi / \Pi^{\prime}$ is cyclic and $Z\left[\zeta_{m}\right]$ can be regarded as a $\Pi / \Pi^{\prime}$-module. $Z \Pi / \Pi^{\prime} \bigotimes_{Z} \mathfrak{a}$ is a quasi-permutation $\Pi / \Pi^{\prime}$-module and $Z\left[\zeta_{m}\right] \mathfrak{a} \cong Z\left[\zeta_{m}\right] \cdot\left(Z \Pi / \Pi^{\prime} \otimes \mathfrak{Z} \mathbb{a}\right)$. Hence we may assume that $\Pi$ is cyclic. In this case we have $\mathfrak{a}^{\Phi_{m}} \oplus S^{\Phi_{m}} \cong S^{\Phi_{m}}$, and therefore $Z\left[\zeta_{m}\right] \cong \mathfrak{a}^{\Phi_{m}} \cong \mathfrak{a}_{\Phi_{m}} \cong Z\left[\zeta_{m}\right] \mathfrak{a}$ by (2.1) and (2.2). This proves the only if part.

Conversely suppose that $\Omega_{\Pi} \mathfrak{a} \cong \Omega_{\Pi}$ as $\Omega_{\Pi}$-modules. Then, by virtue of (2.4), $\mathfrak{a} \oplus \Omega_{\Pi} \cong Z \Pi \oplus \Omega_{\Pi}$. Further, by (2.3), $\Omega_{\Pi}$ is a quasi-permutation $\Pi$-module. Therefore applying (1.4), we can conclude that $a$ is a quasi-permutation $\Pi$ module. Thus the proof of the theorem is completed.

COROLLARY 2.6. Let $\Pi$ be a finite abelian group and let $\Omega_{\Pi}=\sum_{i=1}^{s} \oplus \Omega_{i}$ be the decomposition of $\Omega_{\Pi}$ into Dedekind domains. Then a $Z$-free $\Omega_{\Pi}$-module $M$ is a quasi-permutation $\Pi$-module if and only if $M \cong \sum_{i=1}^{s} \oplus \Omega_{i}^{\left(t_{i}\right)}$ for $t_{i} \geqq 0$.

PROOF. The if part of the corollary follows immediately from (2.3). Suppose that $M$ is a quasi-permutation $\Pi$-module. Since each $\Omega_{i}$ is a Dedekind domain and a quasi-permutation $I I$-module $((2.3))$, we may suppose that $M \cong \sum_{i=1}^{s} \oplus \mathfrak{a}_{i}$ where each $\mathfrak{a}_{i}$ is a non-zero ideal of $\Omega_{i}$. The natural homomorphism $C(Z \Pi) \rightarrow C\left(\Omega_{\Pi}\right)$ is an epimorphism. Therefore there exists a projective ideal a of $Z \Pi$ such that $\Omega_{\Pi} \mathfrak{a} \cong M$. According to (2.4), we have $\mathfrak{a} \oplus \Omega_{\Pi}$ $\cong Z \Pi \oplus M$, and it follows from (2.3) that $\mathfrak{a}-\frac{{ }_{(r)}}{-{ }_{(r)}} 0$. Hence $\mathfrak{a}$ is a quasipermutation $\Pi$-module. By virtue of (2.5) we can conclude that $M \cong \Omega_{I} \mathfrak{a} \cong \Omega_{I}$, which completes the proof of the only if part.

REMARK 2.7. Let $\Pi$ be a finite abelian group and let $\Omega_{\Pi}=\sum_{i=1}^{s} \oplus \Omega_{i}$ be the decomposition of $\Omega_{\Pi}$ into Dedekind domains. Let $P$ be a projective $\Pi$ module. Then we have $P-\frac{(r)}{s} \sum_{i=1}^{s} \oplus \Omega_{i} P$. Especially, if $\Pi$ is cyclic, we have 
$P=\sum_{i=1}^{s} \oplus \Omega_{i} P$

PROOF. The first part follows directly from (2.3) and (2.4), and the second part can be proved along the same line as in the proof of [2], (1.11).

$\S 3$. We sketch the Jacobinski-Roiter's results on orders which will be used in $\S \S 3$ and 4 .

Let $K$ be an algebraic number field and $R$ be the ring of all algebraic integers in $K$. Let $\Sigma$ be a semi-simple $K$-algebra and $\Lambda$ be an $R$-order in $\Sigma$. A $\Lambda$-module is called a $\Lambda$-lattice if it is a finitely generated projective $R$ module and we denote by $\boldsymbol{C}_{A}$ the class of all $A$-lattices. Given $M, N \in \boldsymbol{C}_{A}$, we write $M \sim N$ if, for any prime ideal $\mathfrak{p}$ of $R, M_{\mathfrak{p}} \cong N_{\mathfrak{p}}$ as $\Lambda_{\mathfrak{p}}$-modules.

We say that a $\Lambda$-lattice $M$ satisfies $(\varepsilon)$ if $\operatorname{End}_{\Sigma}(K M)$ does not have any totally definite quaternion algebra as its simple component.

Let $\Omega$ be a maximal $R$-order in $\Sigma$ which contains $\Lambda$. Given $M, N \in C_{A}$ we write $M \approx N$ if $M \sim N$ and $\Omega M \cong \Omega N$ as $\Omega$-modules. We put $\gamma_{M}=\left\{N \in C_{A} \mid\right.$ $N \approx M\}$ and denote by $\left|\gamma_{M}\right|$ the number of all isomorphism types in $\gamma_{M}$. If $M$ satisfies $(\varepsilon)$, then $\gamma_{M}$ does not depend on the choice of $\Omega$.

(A) ([6]). If $N$ is a local direct summand of $M$, then there is a decomposition $M \cong N^{\prime} \oplus L$ with $N^{\prime} \sim N$.

(B) ([6]). If $M$ is $\Lambda$-faithful and $N \sim N^{\prime}$, then there is $M^{\prime}$ such that $M \oplus N \cong M^{\prime} \oplus N^{\prime}$.

(C) ([6]). Suppose that $N$ is a local direct summand of $M$ and that every simple $\Sigma$-module $S$ which occurs in $K M$ occurs strictly more times in $K M$ than in $K N$. Then $N$ is a direct summand of $M$.

(D) ([3]). Let $M$ satisfy $(\varepsilon)$ and $X$ be a local direct summand of $M^{(l)}$ for some l. Then $X \oplus M \cong X \oplus N$ implies $M \cong N$.

(E) ([3]). (i) If $M$ is an $\Omega$-lattice which satisfies $(\varepsilon)$, we have $\left|\gamma_{M}\right|=1$ as a A-lattice. (ii) Let $T$ be a A-faithful, $\Lambda$-lattice satisfying $(\varepsilon)$ such that $\left|\gamma_{T}\right|=1$. Then $M \approx N$ if and only if $M \oplus T \cong N \oplus T$.

$\left(\mathrm{E}^{\prime}\right)([3])$. Let $M$ be a A-lattice. Suppose that $\operatorname{End}_{\Sigma}(K M)$ is a commutative field and that End. $(M)$ is the integral closure of $R$ in $\operatorname{End}_{\Sigma}(K M)$. Then $\left|\gamma_{M}\right|=1$ as a A-lattice.

It is noted that $\left(E^{\prime}\right)$ is a special case of $(E)$, (i).

In (2.5) we have shown that $\tilde{C}(Z \Pi)=C^{q}(Z \Pi)$ for any finite abelian group $\Pi$. Here it is natural to ask whether $C^{q}(Z \Pi)$ coincides with $\widetilde{C}(Z \Pi)$ for any finite group $\Pi$ or not. In this section we will prove that $\tilde{C}(Z \Pi)=C^{q}(Z \Pi)$ for some types of finite groups.

Let $\Pi$ be a finite group. Let $Q \Pi=\Sigma_{1} \oplus \Sigma_{2} \oplus \cdots \oplus \Sigma_{t}$ be the decomposition of $Q \Pi$ into simple algebras. Denote by $K_{i}$ the center of $\Sigma_{i}$ and let $R_{i}$ be the ring of all algebraic integers in $K_{i}$. 
A finite group $\Pi$ is said to be of split type $(\operatorname{over} Q)$ if each $\Sigma_{i}$ is a full matrix algebra over $K_{i}$.

Proposition 3.1. Let $\Pi$ be a finite group of split type. Suppose that, for each $i$, there is a quasi-permutation $\Pi$-module $T_{i}$ such that $\operatorname{End}_{\Sigma_{i}}\left(Q T_{i}\right) \cong K_{i}$ and $\operatorname{End}_{z \Pi}\left(T_{i}\right) \cong R_{i}$. Then $\tilde{C}(Z \Pi) \subseteq C^{q}(Z \Pi)$.

Proof. Put $T=\sum_{i=1}^{t} \oplus T_{i}$. Then $T$ is a faithful quasi-permutation $\Pi$ module. By $\left(\mathrm{E}^{\prime}\right)$ we have $\left|\gamma_{T_{i}}\right|=1$ for each $i$. Then, using (A), we easily see that $\left|\gamma_{T}\right|=1$. Let $a$ be a projective ideal of $Z \Pi$ such that $a \approx Z \Pi$. By virtue of (E), (ii) we have $a \oplus T \cong Z \Pi \oplus T$. According to (1.4) this implies that $\mathfrak{a}$ is a quasi-permutation $\Pi$-module, which completes the proof.

Let $S$ be a Dedekind domain with quotient field $L$. Let $\Pi$ be a finite group of automorphisms of $L$ and put $K=L^{I}$ and $R=S^{I}$. We denote by $\Delta(\Pi, S)(\Delta(\Pi, L))$ the twisted group algebra of $\Pi$ over $S(L)$. Then $\Delta(\Pi, L)$ is isomorphic to a full matrix algebra over $K$ and $\Delta(\Pi, S)$ can be regarded as an $R$-order in $\Delta(\Pi, L)$.

Especially, if $S / R$ is tamely ramified, then $\Delta(\Pi, S)$ is hereditary, as is well known, and any finitely generated projective $\Delta(\Pi, S)$-module is expressible as a direct sum of ambiguous ideals of $S$ (cf. [8]).

LEMMA 3.2. Suppose that $S / R$ is tamely ramified. Then $\left|\gamma_{\Delta(\pi, S)}\right|=1$.

Proof. Let $\mathfrak{a}$ be an ambiguous ideal of $S$. Then we have $\operatorname{End}_{\Delta(\pi, S)} \mathfrak{a} \cong$ $S^{I}=R$, hence, by $\left(\mathrm{E}^{\prime}\right),\left|\gamma_{a}\right|=1$. Now we can write $\Delta(\Pi, S) \cong \sum_{i=1}^{l} \oplus \mathfrak{a}_{i}$ as $\Delta(I, S)$-modules where each $\mathfrak{a}_{i}$ is an ambiguous ideal of $S$. Hence, using (C), we easily see that $\left|\gamma_{\Delta(\pi, S)}\right|=1$.

LEMMA 3.3. Let $\Pi$ be a finite group. Suppose that there is an order $\Lambda$ in $Q \Pi$ containing $Z \Pi$ which is a quasi-permutation $\Pi$-module with $\left|\gamma_{\Lambda}\right|=1$. Then $\tilde{C}(Z \Pi) \subseteq C^{q}(Z \Pi)$.

Proof. This follows directly from (2.4) (or (E)) and (1.4).

We denote by $\Pi_{n, m, r}$ the metacyclic group with generators $\sigma$ and $\tau$ satisfying the relations:

$$
\tau^{-1} \sigma \tau=\sigma^{r}, \quad \sigma^{n}=\tau^{m}=I
$$

where $(r, n)=1$ and $r^{m} \equiv 1 \bmod n$. It is remarked that the group $\Pi_{n, 2,-1}$ means the dihedral group $D_{n}$ of order $2 n$.

PROPOSITION 3.4. Let $\Pi$ be one of the following groups:

(1) nilpotent groups of odd order;

(2) metacyclic groups $\left\{\Pi_{n, q, r}\right\}$ where $q$ is a prime such that $q+n$;

(3) dihedral groups $\left\{D_{n}\right\}$;

(4) the alternating group $A_{4}$ and the symmetric group $S_{4}$. Then $\tilde{C}(Z \Pi) \subseteq C^{q}(Z \Pi)$.

Proof. (1) Let $\Pi$ be a finite nilpotent group of odd order $n$. Let 
$Q \Pi=\Sigma_{1} \oplus \Sigma_{2} \oplus \cdots \oplus \Sigma_{t}$ be the decomposition of $Q \Pi$ into simple algebras and denote by $K_{i}$ the center of $\Sigma_{i}$. By the well-known Witt-Roquette's theorem ([7]), for each $i$, the simple algebra $\Sigma_{i}$ is a full matrix algebra over $K_{i}$ and the field $K_{i}$ is a cyclotomic field $Q\left(\zeta_{n_{i}}\right)$ for some $n_{i} \mid n$. Further let $V_{i}$ be a simple $\Sigma_{i}$-module and let $\chi_{i}$ be the character of $\Pi$ afforded by $V_{i}$. Then there exist a subgroup $\Pi_{i}$ of $\Pi$ and a one dimensional $K_{i} \Pi_{i}$-module $K_{i}$ with character $\rho_{i}$ such that $V_{i} \cong Q \Pi \underset{Q \Pi_{i}}{\otimes} K_{i}$ and $Q\left(\chi_{i}\right)=Q\left(\rho_{i}\right)=K_{i}$. Now we put $T_{i}=Z \Pi \otimes_{Z \Pi_{i}} Z\left[\zeta_{n_{i}}\right]$. Then we see that $\operatorname{End}_{z \Pi}\left(T_{i}\right) \cong Z\left[\zeta_{n_{i}}\right]$, and from (2.3) it follows that each $T_{i}$ is a quasi-permutation $\Pi$-module. So we have $\widetilde{C}(Z \Pi) \subseteq$ $C^{q}(Z I)$ from (3.1).

(2) Let $\Pi=\Pi_{n, q, r}$ where $q$ is a prime such that $q+n$. Put $\Pi_{0}=[\tau]$, $\Pi_{1}=[\sigma]$ and $m=(r-1, n)$. We can write $Q \Pi=\sum_{l \mid n} \oplus Q \Pi /\left(\Phi_{l}(\sigma)\right)$. If $l$ divides $m$, then $\Pi /\left[\sigma^{l}\right]$ is a cyclic group and therefore we have $Q \Pi /\left(\Phi_{l}(\sigma)\right) \cong Q\left(\zeta_{l}\right)$ $\oplus Q\left(\zeta_{q l}\right)$. Then the images of $Z I I$ by the projections on $Q\left(\zeta_{l}\right)$ and $Q\left(\zeta_{q l}\right)$ are $Z\left[\zeta_{l}\right]$ and $Z\left[\zeta_{q l}\right]$, respectively. Since both $Z\left[\zeta_{l}\right]$ and $Z\left[\zeta_{q l}\right]$ are regarded as $\Pi /\left[\sigma^{l}\right]$-modules, according to (2.3) these are quasi-permutation $\Pi$-modules and, by $\left(E^{\prime}\right),\left|\gamma_{z\left[\zeta_{l}\right]}\right|=\left|\gamma_{z\left[\left[_{q l}\right]\right.}\right|=1$. On the other hand, if $l$ does not divide $m$, then $Q \Pi /\left(\Phi_{l}(\sigma)\right)$ is isomorphic to the twisted group algebra $\Delta\left(\Pi_{0}, Q\left(\zeta_{l}\right)\right)$ because $q$ is a prime and the order $Z \Pi /\left(\Phi_{l}(\sigma)\right)$ in $Q \Pi /\left(\Phi_{l}(\sigma)\right)$ is also isomorphic to the twisted group algebra $\Delta\left(\Pi_{0}, Z\left[\zeta_{l}\right]\right)$. From the assumption that $q$ is a prime such that $q+n$ it is easily seen that $Z\left[\zeta_{l}\right] / Z\left[\zeta_{l}\right]^{I_{0}}$ is tamely ramified, and hence, by (3.2), $\left|\gamma_{\Delta\left(\Pi_{0}, Z\left[\zeta_{l}\right]\right)}\right|=1$. It is clear that $\Delta\left(\Pi_{0}, Z\left[\zeta_{l}\right]\right) \cong Z \Pi_{Z \Pi_{1}} Z\left[\zeta_{l}\right]$ as $\Pi$-modules and so, by (2.3), $\Delta\left(\Pi_{0}, Z\left[\zeta_{l}\right]\right)$ is a quasi-permutation $\Pi$-module. We put $\Lambda=\sum_{l l m} \oplus\left(Z\left[\zeta_{l}\right] \oplus Z\left[\zeta_{q l}\right]\right) \oplus \sum_{l \neq m} \oplus \Delta\left(\Pi_{0}, Z\left[\zeta_{l}\right]\right)$. Then $\Lambda$ is a hereditary order in $Q \Pi$ containing $Z \Pi$ and a quasi-permutation $\Pi$-module such that $\left|\gamma_{\Lambda}\right|=1$. Hence, from (3.3), we get $\tilde{C}(Z \Pi) \subseteq C^{q}(Z \Pi)$.

(3) Let $\Pi=D_{n}=\Pi_{n, 2,-1}$. When $2+n$ this is a special case of (2). Hence we have only to prove the assertion when $2 \mid n$. We can write $Q \Pi=$ $\sum_{l \mid n} \oplus Q \Pi /\left(\Phi_{l}(\sigma)\right)$. Here $Q \Pi /\left(\Phi_{1}(\sigma)\right) \cong Q \oplus Q \Pi /(\sigma-1, \tau+1)$ and $Q \Pi /\left(\Phi_{2}(\sigma)\right) \cong$ $Q \Pi /(\sigma+1, \tau-1) \oplus Q \Pi /(\sigma+1, \tau+1)$. For each $l \mid n, l>2, Q \Pi /\left(\Phi_{l}(\sigma)\right)$ is isomorphic to the twisted group algebra $\Delta\left(\Pi_{0}, Q\left(\zeta_{l}\right)\right)$ and the order $Z \Pi /\left(\Phi_{l}(\sigma)\right)$ in $Q \Pi /\left(\Phi_{l}(\sigma)\right)$ is also isomorphic to the twisted group algebra $\Delta\left(\Pi_{0}, Z\left[\zeta_{l}\right]\right)$. We put $T^{(1)}=Z, \quad T^{(2)}=Z \Pi /(\sigma-1, \tau+1), \quad T^{(3)}=Z \Pi /(\sigma+1, \tau-1)$ and $T^{(4)}=$ $Z \Pi /(\sigma+1, \tau+1)$. Then it is clear that $T^{(i)}$ is a quasi-permutation $\Pi$-module with $\left|\gamma_{T^{(i)}}\right|=1$. Further, putting $T_{l}=\Delta\left(\Pi_{0}, Z\left[\zeta_{l}\right]\right)(\tau-1)=Z \Pi /\left(\Phi_{l}(\sigma), \tau+1\right)$ for any $l \mid n, l>2$, we can show using the same method as in (2.3) that $T_{l}$ is a quasi-permutation $\Pi$-module. We easily see that $\operatorname{End}_{z \Pi}\left(T_{l}\right)=Z\left[\zeta_{l}+\zeta_{l}^{-1}\right]=$ $Z\left[\zeta_{l}\right]^{\Pi_{0}}$, and therefore, by $\left(\mathrm{E}^{\prime}\right)$, we have $\left|\gamma_{T_{l}}\right|=1$. Thus we conclude by (3.1) that $\tilde{C}(Z \Pi) \subseteq C^{q}(Z \Pi)$. 
(4) Both $A_{4}$ and $S_{4}$ are of split type, as is well known. The assertion can be proved using (3.1).

To show the inverse inclusion $C^{q}(Z \Pi) \subseteq \widetilde{C}(Z \Pi)$ we must refer to a Conlon's result.

LemMA 3.5 ([1]). Let $\Pi$ be a finite group of order $n$. Suppose that $\sum_{i=1}^{s} \oplus Z \Pi / \Pi_{i}^{\prime} \oplus L \cong \sum_{j=1}^{t} \oplus Z \Pi / \Pi_{j}^{\prime \prime} \oplus L$ where $\Pi_{i}^{\prime}$ and $\Pi_{j}^{\prime \prime}$ are subgroups of $\Pi$ each of which is a cyclic extension of a p-subgroup of $\Pi$ for some prime $p \mid n$ and $L$ is a $\Pi$-module. Then $s=t$ and the $\Pi_{j}^{\prime \prime}$ can be reordered so that $Z \Pi / \Pi_{i}^{\prime} \cong$ $Z \Pi / \Pi_{j}^{\prime \prime}$ for any $1 \leqq i \leqq s$.

Proposition 3.6. Let $\Pi$ be a finite group which is a cyclic extension of a p-group. Then $C^{q}(Z \Pi) \subseteq \tilde{C}(Z \Pi)$. Further let $\subseteq$ be a complete set of nonconjugate subgroups of $\Pi$, and put $T=\sum_{\Pi^{\prime} \in \subseteq} \oplus Z \Pi / \Pi^{\prime}$ in the case where no simple component of $Q \Pi$ is a totally definite quaternion algebra and $T=$ $Z \Pi \bigoplus_{\Pi^{\prime} \in \Subset} \oplus Z \Pi / \Pi^{\prime}$ in the other case. Then, for any quasi-permutation projective ideal a of $Z \Pi, \mathfrak{a} \oplus T \cong Z \Pi \oplus T$, and $\left|\gamma_{T}\right|=\left[\widetilde{C}(Z \Pi): C^{q}(Z \Pi)\right]$.

ProOF. Let $a$ be a quasi-permutation projective ideal of $Z \Pi$. Then we have $a \oplus \sum_{i=1}^{s} \oplus Z \Pi / \Pi_{i}^{\prime} \cong \sum_{j=1}^{t} \oplus Z \Pi / \Pi_{j}^{\prime \prime}$ for some subgroups $\Pi_{i}^{\prime}$, $\Pi_{j}^{\prime \prime}$ of $\Pi$. However $\mathfrak{a}^{(k)} \cong Z \Pi^{(k)}$ for some $k>0$ because $C(Z \Pi)$ is a finite group ([11]). Hence $Z \Pi^{(k)} \oplus \sum_{i=1}^{s} \oplus\left(Z \Pi / \Pi_{i}^{\prime}\right)^{(k)} \cong \sum_{j=1}^{t} \oplus\left(Z \Pi / \Pi_{j}^{\prime \prime}\right)^{(k)}$. By (3.5) we have $s=t$ and $\Pi_{0}^{\prime \prime}=I, Z \Pi / \Pi_{i}^{\prime \prime} \cong Z \Pi / \Pi_{i}^{\prime}$ for any $1 \leqq i \leqq s$ by reordering the $\Pi_{j}^{\prime \prime}$. Therefore $\mathfrak{a} \oplus \sum_{i=1}^{s} \oplus Z \Pi / \Pi_{i}^{\prime} \cong Z \Pi \oplus \sum_{i=1}^{s} \oplus Z \Pi / \Pi_{i}^{\prime}$. From this and (D) we get $\Omega_{\Pi} \mathfrak{a} \oplus \Omega_{\Pi}$ $\cong \Omega_{\Pi} \oplus \Omega_{\Pi}$ where $\Omega_{\Pi}$ denotes a maximal order in $Q \Pi$ containing $Z \Pi$. This shows that $C^{q}(Z \Pi) \subseteq \widetilde{C}(Z \Pi)$. By (D) we have also $\mathfrak{a} \oplus T \cong Z \Pi \oplus T$. Since $T$ is $Z \Pi$-faithful, we easily see using (B) and (D) that $\left|\gamma_{T}\right|=\left[\tilde{C}(Z \Pi): C^{q}(Z \Pi)\right]$.

REMARK 3.7. Let $\Pi$ be a finite group of split type. Let $F=K_{1} \oplus K_{2}$ $\oplus \cdots \oplus K_{t}$ be the center of $Q \Pi$. Suppose that, for each $i$, the class number of $K_{i}$ is 1 . Then $C^{q}(Z \Pi) \subseteq \widetilde{C}(Z \Pi)=C(Z \Pi)$.

Proof. Since $\Pi$ is of split type, we have $C\left(\Omega_{\Pi}\right)=C(F)([5])$. By the assumption, $C(F)=\sum_{i=1}^{t} \oplus C\left(K_{i}\right)=0$, hence $C\left(\Omega_{I}\right)=0$. Thus $\tilde{C}(Z \Pi)=C(Z \Pi)$.

We denote by $H_{n}(n \geqq 2)$ the generalized quaternion group of order $4 n$, i. e., the group with generators $\sigma$ and $\tau$ satisfying the relations:

$$
\sigma^{2 n}=I, \quad \sigma^{n}=\tau^{2}, \quad \tau^{-1} \sigma \tau=\sigma^{-1} .
$$

Let $N_{n}=\left[\sigma^{n}\right]=\left[\tau^{2}\right]$ and $H_{n}^{\prime}=[\sigma]$. Then $H_{n} / N_{n} \cong D_{n}$ and $Q H_{n} \cong Q D_{n} \oplus$ $Q H_{n} /\left(\sigma^{n}+I\right)$. Let $n=2^{m_{n}} n_{0}, 2+n_{0}$. Then $Q H_{n} /\left(\sigma^{n}+I\right) \cong \sum_{2^{m_{l} l \mid} n} \oplus Q H_{n} /\left(\Phi_{2 l}(\sigma)\right)$. For any $2^{m}|l| n, l>1, \Sigma_{l}=Q H_{n} /\left(\Phi_{2 l}(\sigma)\right)$ is a quaternion algebra over $Q\left(\zeta_{2 l}+\zeta_{2 l}^{-1}\right)$. 
In case $n$ is odd there is a simple component $Q H_{n} /\left(\Phi_{2}(\sigma)\right) \cong Q(i)$ in $Q H_{n} /\left(\sigma^{n}+I\right)$. For each $2^{m}|l| n, Z H_{n} /\left(\Phi_{2 l}(\sigma)\right) \cong Z H_{n} \underset{Z H_{n}^{\prime}}{\otimes} Z\left[\zeta_{2 l}\right]$ and so $\Lambda_{l}=Z H_{n} /\left(\Phi_{2 l}(\sigma)\right)$ is a quasi-permutation $H_{n}$-module. Let $\Omega_{D_{n}}$ be a maximal order in $Q D_{n}$ which contains $Z D_{n}$. We put $\Lambda_{H_{n}}=\Omega_{D_{n}} \oplus \sum_{2^{m_{|l|} n}} \oplus \Lambda_{l}$. Then $\Lambda_{H_{n}}$ is an order in $Q H_{n}$ which contains $Z H_{n}$. Let $\Omega_{H_{n}}$ be a maximal order in $Q H_{n}$ which contains $\Lambda_{H_{n}}$. There are natural epimorphisms $\alpha_{n}: C\left(Z H_{n}\right) \rightarrow C\left(\Lambda_{H_{n}}\right)$ and $\beta_{n}: C\left(\Lambda_{H_{n}}\right) \rightarrow$ $C\left(\Omega_{H_{n}}\right)$ induced by $\Lambda_{H_{n}} \underset{2 H_{n}}{\otimes} \cdot$ and $\Omega_{H_{n}} \otimes \cdot$, respectively.

Proposition 3.8. For any prime power $p^{l}$ there is an exact sequence:

$$
0 \longrightarrow C^{q}\left(Z H_{p l}\right) \longrightarrow C\left(Z H_{p l}\right) \stackrel{\alpha_{p l}}{\longrightarrow} C\left(\Lambda_{H p l}\right) \longrightarrow 0 .
$$

We have $\tilde{C}\left(Z H_{p l}\right)=C^{q}\left(Z H_{p l}\right)$ if and only if $\beta_{p^{l}}: C\left(\Lambda_{H_{p l}}\right) \rightarrow C\left(\Omega_{H_{p l} l}\right)$ is an isomorphism. Especially $C\left(\Lambda_{H_{2}}\right)=0$ and $C^{q}\left(Z H_{2}\right)=\tilde{C}\left(Z H_{2}\right)=C\left(Z H_{2}\right)$.

Proof. Let $a$ be a quasi-permutation projective ideal of $Z H_{p l}$. Then $\mathfrak{a} /\left(\sigma^{p l}-1\right) \mathfrak{a}$ is also a quasi-permutation projective ideal of $Z D_{p l}$. By (3.4) and (3.6) we have $\tilde{C}\left(Z D_{p l}\right)=C^{q}\left(Z D_{p l}\right)$. Hence we see $\Omega_{D_{p l}} \mathfrak{a} \cong \Omega_{D p l}$. On the other hand, since each $\Sigma_{p m}$ is a division algebra, for any subgroup $H$ of $H_{p l}$, $\Lambda_{p m} \cdot Z H_{p l} / H \cong \Lambda_{p m}$ or 0 . From this it follows that $\Lambda_{p m} \mathfrak{a} \oplus \Lambda_{p m} \cong \Lambda_{p m} \oplus \Lambda_{p m}$. Thus we have $\Lambda_{H p l} \mathfrak{a} \oplus \Lambda_{H p l} \cong \Lambda_{H p l} \oplus \Lambda_{H p l}$. Conversely let $\mathfrak{a}$ be a projective ideal of $Z H_{p l}$ such that $\Lambda_{H p l} \mathfrak{a} \oplus \Lambda_{H p l} \cong \Lambda_{H p l} \oplus \Lambda_{H p l}$. Then $\Omega_{H p l} \mathfrak{a} \cong \Omega_{H p l}$ and so $\mathfrak{a} /\left(\sigma^{p l}-1\right) \mathfrak{a}$ is a quasi-permutation $H_{p l}$-module. We have also that, for any $m \leqq l, \Lambda_{p m} \mathfrak{a} \oplus \Lambda_{p m} \cong \Lambda_{p m} \oplus \Lambda_{p m}$. Since $\Lambda_{p m}$ is a quasi-permutation $H_{p l}$-module, according to (1.4), $\Lambda_{p m} \mathfrak{a}$ is also a quasi-permutation $H_{p l}$-module. Using the same method as in (2.3) we can show that $\mathfrak{a} /\left(\sigma^{p l}+1\right) \mathfrak{a}$ is a quasi-permutation $H_{p l}$-module. Furthermore, considering an exact sequence:

$$
0 \longrightarrow \mathfrak{a} /\left(\sigma^{p l}+1\right) \mathfrak{a} \longrightarrow \mathfrak{a} \longrightarrow \mathfrak{a} /\left(\sigma^{p l}-1\right) \mathfrak{a} \longrightarrow 0,
$$

it can easily be seen that $a$ is a quasi-permutation $H_{p l}$-module. This completes the proof of Kernel $\alpha_{p l}=C^{q}\left(Z H_{p l}\right)$. The second part of the proposition is obvious. Hence we will prove only $C\left(\Lambda_{H_{2}}\right)=0$. We have $\Lambda_{H_{2}}=\Omega_{D_{2}} \oplus \Lambda_{2}$, and therefore it suffices to show that any projective ideal of $\Lambda_{2}$ is principal. The quaternion algebra $\Sigma_{2}$ is generated by $i, j, k$ over $Q$ such that $i^{2}=j^{2}=-1$, $i j=-j i$ and $k=i j$ and $\Lambda_{2}$ can be identified with the order $Z+Z i+Z j+Z k$ in $\Sigma_{2}$. Let $\Gamma$ be the order in $\Sigma_{2}$ generated by $i, j, k, \frac{1}{2}(1+i+j+k)$. Then, as is well known, $\Gamma$ is a maximal order in $\Sigma_{2}$ which contains $\Lambda_{2}$ and the class number $c(\Gamma)$ of $\Gamma$ is 1 . Let $\mathfrak{a}$ be a projective ideal of $\Lambda_{2}$. Then there is an ideal $\mathfrak{b}$ of $\Lambda_{2}$ such that $\mathfrak{a} \cong \mathfrak{b}$ and $2 \nmid\left[\Lambda_{2}: \mathfrak{b}\right]$. Therefore we may suppose $2+\left[\Lambda_{2}: \mathfrak{a}\right]$. Since $\left[\Gamma: \Lambda_{2}\right]=2$, we have $\mathfrak{a}=\Gamma \mathfrak{a} \cap \Lambda_{2}$. Because of $c(\Gamma)=1$ there exists $\omega \in \Gamma$ such that $\Gamma \mathfrak{a}=\Gamma \omega$. Then the norm of $\omega$ is odd. Therefore we can find a unit $\varepsilon$ of $\Gamma$ such that $\varepsilon \omega \equiv 1 \bmod 2$ by the well-known result on 
quaternions. Accordingly we can show that $a=\Lambda_{2} \varepsilon \omega, \varepsilon \omega \in \Lambda_{2}$, which completes the proof of the proposition.

We remark here that Reiner-Ullom $([4])$ has proved that $C\left(Z H_{2}\right) \cong Z / 2 Z$.

From (3.4), (3.6), (3.7) and (3.8) we get

THEOREM 3.9. Let $\Pi$ be one of the following groups:

(1) $p$-groups with $p \neq 2$;

(2) metacyclic groups $\left\{\Pi_{p^{l}, q, r}\right\}$ where $p, q$ are distinct primes;

(3) dihedral groups $\left\{D_{p l}\right\}$ where $p$ is a prime;

(4) the quaternion group $H_{2}$, the alternating group $A_{4}$ and the symmetric group $S_{4}$.

Then we have $\tilde{C}(Z \Pi)=C^{q}(Z \Pi)$.

$\S 4$. In this section we will give a basic result on projective quasipermutation modules over a finite abelian group which is a refinement of (2.5).

LEMMA 4.1. Let $\Pi=[\sigma]$ be a cyclic group of order $n$ and $\mathfrak{a}$ be a projective ideal of $Z \Pi$ such that $a^{\Phi_{n}} \cong Z\left[\zeta_{n}\right]$. Then there exist a projective ideal $\mathrm{c}$ of $Z \Pi$ with $c^{\phi_{l}} \cong Z\left[\zeta_{l}\right]$ for any $l \mid n$, a permutation $\Pi$-module $S$ and a projective ideal $\mathfrak{a}_{m}$ of $Z \Pi /\left[\sigma^{m}\right]$ for any $m \mid n$ such that $n / m$ is a prime, such that

$$
\mathfrak{a} \oplus \sum_{m} \oplus Z \Pi /\left[\sigma^{m}\right] \oplus S \cong \mathfrak{c} \oplus \sum_{m} \oplus \mathfrak{a}_{m} \oplus S .
$$

Proof. Let $M_{1}=\mathfrak{a} \oplus \sum_{m} \oplus\left[\mathfrak{a} /\left(\sigma^{m}-1\right) \mathfrak{a}\right]^{-1}$. Then $M_{1}^{\phi_{n}}=\mathfrak{a}^{\phi_{n}}$ and $M_{1}^{\Phi_{m}}=$ $\mathfrak{a}^{\Phi_{m}} \oplus\left(\mathfrak{a}^{-1}\right)^{\Phi_{m}} \cong Z\left[\zeta_{m}\right]^{(2)}$ for any $m \mid n$ such that $n / m$ is a prime. Also, for any $l \mid n$ with $n / l=p_{1} p_{2}$ for primes $p_{1}, p_{2}, M_{1}^{\phi_{l}} \cong Z\left[\zeta_{l}\right]^{(2)} \oplus\left[\left(\mathfrak{a}^{-1}\right)^{\phi_{l}}\right]^{\left(\alpha_{l}\right)}$ for some $\alpha_{l} \geqq 0$. We now put $M_{2}=M_{1} \oplus_{n / l=p_{1} p_{2}} \oplus\left[\mathfrak{a} /\left(\sigma^{l}-1\right) \mathfrak{a}\right]^{\left(\alpha_{l}\right)}$. Then, by (2.1), we have

$$
\begin{aligned}
& M_{2}^{\Phi_{n}} \cong Z\left[\zeta_{n}\right], \\
& M_{2}^{\Phi_{m}} \cong Z\left[\zeta_{m}\right]^{(2)} \quad \text { when } n / m=p, \\
& M_{2}^{\Phi_{l}} \cong Z\left[\zeta_{l}\right]^{\left(2+2 \alpha_{l}\right)} \quad \text { when } n / l=p_{1} p_{2},
\end{aligned}
$$

and

$$
M_{2}^{\Phi_{k}} \cong Z\left[\zeta_{k}\right]^{\left(\beta_{k}\right)} \oplus\left[\left(\mathfrak{a}^{ \pm 1}\right)^{\Phi_{k}}\right]^{\left(\gamma_{k}\right)} \quad \text { for some } \quad \beta_{k}, \gamma_{k} \geqq 0
$$

when $n / k=p_{1} p_{2} p_{3}$. We further put

$$
M_{3}=M_{2} \oplus \sum_{n / k=p_{1} p_{2} p_{3}} \oplus\left[\left(\mathfrak{a} /\left(\sigma^{k}-1\right) \mathfrak{a}\right)^{\mp 1}\right]^{\left(\gamma_{k}\right)}
$$

and repeat the same procedure to $M_{3}$ as to $M_{2}$. Continuing this procedure, we finally find $s_{l} \geqq 0$ and $\varepsilon(l)= \pm 1$ for any $l \mid n$ such that, putting $M=$ $\mathfrak{a} \oplus \sum_{\substack{l i n \\ l<\mathfrak{n}}} \oplus\left[\left(\mathfrak{a} /\left(\sigma^{l}-1\right) \mathfrak{a}\right)^{\varepsilon(l)}\right]^{(s l)}, M^{\Phi_{k}}$ is $Z\left[\zeta_{k}\right]$-free for any $k \mid n$. Since $\mathfrak{a}$ is $Z \Pi$. faithful and $\mathfrak{a} /\left(\sigma^{l}-1\right) \mathfrak{a} \sim Z \Pi /\left[\sigma^{l}\right]$, by $(\mathrm{C})$, there is a projective ideal of $Z \Pi$ 
such that $M \cong \mathfrak{c} \oplus \sum_{\substack{l, n \\ l<n}} \oplus\left[Z \Pi /\left[\sigma^{l}\right]\right]^{(s l)}$. Then we see easily that $c^{\Phi_{k}} \cong Z\left[\zeta_{k}\right]$ for any $k \mid n$. Therefore $c$ is as required. Furthermore we have

$$
\begin{aligned}
M \oplus \sum_{\substack{l \backslash n \\
l<n}} \oplus\left[\left(\mathfrak{a} /\left(\sigma^{l}-1\right) \mathfrak{a}\right)^{-\varepsilon(l)}\right]^{\left(s_{l}\right)} & \cong \mathfrak{a} \oplus \sum_{\substack{l>n \\
l<n}} \oplus\left[Z \Pi /\left[\sigma^{l}\right]\right]^{\left(2 s_{l}\right)} \\
& \cong \mathfrak{c} \oplus \sum_{\substack{l \backslash n \\
l<n}} \oplus\left[Z \Pi /\left[\sigma^{l}\right] \oplus\left(\mathfrak{a} /\left(\sigma^{l}-1\right) \mathfrak{a}\right)^{-\varepsilon(l)}\right]^{\left(s_{l}\right)} .
\end{aligned}
$$

Again, by (C), there is a $\Pi$-module $L$ such that

$$
L \sim \sum_{n / m=p} \oplus Z \Pi /\left[\sigma^{m}\right]
$$

and

$$
\begin{aligned}
& \sum_{\substack{l, n \\
l<n}} \oplus\left[Z \Pi /\left[\sigma^{l}\right] \oplus\left(\mathfrak{a} /\left(\sigma^{l}-1\right) \mathfrak{a}\right)^{-\varepsilon(l)}\right]^{\left(s_{l}\right)} \\
& \cong L \oplus \sum_{n / m=p} \oplus\left[Z \Pi /\left[\sigma^{m}\right]\right]^{\left(2 s_{m^{-1}}\right)} \oplus \sum_{\substack{n / l=p_{1} p_{2} \cdots p_{t} \\
t \geq 2}} \oplus\left[Z \Pi /\left[\sigma^{l}\right]\right]^{\left(2 s_{l}\right)} .
\end{aligned}
$$

Using (A), we can write $L \cong \sum_{n / m=p} \oplus \mathfrak{a}_{m}$ for some $\mathfrak{a}_{m} \sim Z \Pi /\left[\sigma^{m}\right]$. Let

$$
S=\sum_{n / m=p} \oplus\left[Z \Pi /\left[\sigma^{m}\right]\right]^{\left(2 s_{m}-1\right)} \oplus \sum_{\substack{n / l=p_{1} p_{2} \cdots p_{t} \\ t \geqq 2}} \oplus\left[Z \Pi /\left[\sigma^{l}\right]\right]^{\left(2 s_{l}\right)} .
$$

Then we obtain

$$
\mathfrak{a} \oplus \sum_{n / m=p} \oplus\left[Z \Pi /\left[\sigma^{m}\right]\right] \oplus S \cong \mathfrak{c} \oplus \sum_{n / m=p} \oplus \mathfrak{a}_{m} \oplus S,
$$

and this completes the proof of the lemma.

THEOREM 4.2. Let $\Pi$ be a finite abelian group and $\mathfrak{a}$ be a projective ideal of $Z \Pi$. Then a is a quasi-permutation $\Pi$-module if and only if $\mathfrak{a} \oplus \sum_{\Pi^{\prime} \in \mathfrak{S}} \oplus Z \Pi / \Pi^{\prime} \cong$ $Z \Pi \oplus \sum_{\Pi^{\prime} \in \subseteq} \oplus Z \Pi / \Pi^{\prime}$, where $\mathfrak{S}$ denotes the set of all subgroups, $\Pi^{\prime}(\neq 1)$, of $\Pi$ such that $\Pi / \Pi^{\prime}$ is cyclic.

Proof. The if part is evident. Hence we have only to prove the only if part. Suppose that $\mathfrak{a}$ is a quasi-permutation $\Pi$-module. Then $\mathfrak{a} \oplus S^{\prime} \cong S$ for some permutation $\Pi$-modules $S, S^{\prime}$ by (1.6), and $\Omega_{\Pi} \mathfrak{a} \cong \Omega_{\Pi}$ by (2.5), where $\Omega_{I I}$ denotes the maximal order in $Q \Pi$ containing $Z \Pi$.

First suppose that $\Pi$ is a cyclic group of order $n$ with a generator $\sigma$. In this case we can write

$$
S=\sum_{k \mid n} \oplus\left[Z \Pi /\left[\sigma^{k}\right]\right]^{\left(N_{k}\right)}, \quad S^{\prime}=\sum_{k \nmid n} \oplus\left[Z \Pi /\left[\sigma^{k}\right]\right]^{\left(N_{k}^{\prime}\right)}
$$

for some $N_{k}, N_{k}^{\prime}(\geqq 0)$. Since $\mathbb{S}=\left\{\left[\sigma^{k}\right]\right\}_{k \mid n, k<n}$ in this case, it suffices by (D) to prove $N_{n}=N_{n}^{\prime}+1$ and $N_{k}=N_{k}^{\prime}$ for any $k<n$. By our assumption we have $Z\left[\zeta_{n}\right] \oplus S^{\phi_{n}} \cong S^{\Phi_{n}}$. But, by (2.1), $S^{\Phi_{n}} \cong\left[Z\left[\zeta_{n}\right]\right]^{\left(N_{n}^{\prime}\right)}$ and $S^{\Phi_{n}} \cong\left[Z\left[\zeta_{n}\right]\right]^{(N n)}$. Hence $N_{n}=N_{n}^{\prime}+1$. Let $m \mid n, m<n$ and suppose that $N_{n}=N_{n}^{\prime}+1$ and $N_{k}=$ 
$N_{k}^{\prime}$ for each $m<k<n$. Then we have $Z\left[\zeta_{m}\right] \oplus S^{\Phi_{m}} \cong S^{\Phi_{m}}$ and, again applying (2.1),

$$
\begin{aligned}
& S^{\prime \Phi} m \cong\left[Z\left[\zeta_{m}\right]\right]^{\left(N_{n}-1\right)} \oplus \bigoplus_{\substack{m \mid k>n \\
m<k<n}} \oplus\left[Z\left[\zeta_{m}\right]\right]^{\left(N_{k}\right)} \oplus\left[Z\left[\zeta_{m}\right]\right]^{\left(N_{m}^{\prime}\right)} \\
& S^{\Phi_{m}} \cong\left[Z\left[\zeta_{m}\right]\right]^{\left(N_{n}\right)} \oplus \bigoplus_{\substack{m<k>n \\
m<k<n}} \oplus\left[Z\left[\zeta_{m}\right]\right]^{\left(N_{k}\right)} \oplus\left[Z\left[\zeta_{m}\right]\right]^{\left(N_{m}\right)} .
\end{aligned}
$$

This shows $N_{m}=N_{m}^{\prime}$.

Now suppose that $\Pi$ is a non-cyclic abelian group. We denote by $\widetilde{S}_{0}$ the subset of $\widetilde{S}$ consisting of all minimal members of $\mathfrak{S}$. We define $\widetilde{S}_{k+1}$ to be the subset of $\mathfrak{S}$ consisting of $\mathfrak{S}_{k}$ and all minimal elements of $\mathfrak{S}_{-} \mathfrak{S}_{k}$. Then we obtain an ascending chain of the subsets of $\mathfrak{S}: \mathfrak{S}_{0} \subsetneq \mathfrak{S}_{1} \subsetneq \cdots \mathfrak{F}_{t}=\mathfrak{S}$. Let $\widetilde{\mathfrak{S}}_{k}$ be the set of all maximal elements in $\widetilde{S}_{k}$. To prove our assertion it suffices to prove that, for any $0 \leqq k \leqq t$, there exist some $\mathfrak{a}_{\Pi^{\prime}}$ with

$$
\begin{aligned}
& \mathfrak{a}_{\Pi^{\prime}} \sim Z \Pi / \Pi^{\prime}\left(\Pi^{\prime} \in \widetilde{\mathfrak{S}}_{k}\right) \text { such that } \\
& \mathfrak{a} \oplus \sum_{\Pi^{\prime}=\Im_{k}} \oplus Z \Pi / \Pi^{\prime} \bigoplus_{\Pi^{\prime \prime} \in \sum_{-}-\Im_{k}} \oplus Z \Pi / \Pi^{\prime \prime} \\
& \cong Z \Pi \bigoplus_{\Pi^{\prime} \in \mathfrak{\Im}_{k}-\widetilde{\Im}_{k}} \oplus Z \Pi / \Pi^{\prime} \oplus \sum_{\Pi^{\prime} \in \widetilde{\mathfrak{\Im}}_{k}} \oplus \mathfrak{a}_{\Pi^{\prime}} \oplus \sum_{\Pi^{\prime \prime} \in \subseteq_{-}-\Im_{k}} \oplus Z \Pi / \Pi^{\prime \prime}
\end{aligned}
$$

In fact $(*)_{t}$ implies our assertion, because $\widetilde{S}_{t}=\mathbb{S}$ and $\widetilde{\Phi}_{t}=\{\Pi\}$. We will prove $(*)_{k}$ by induction on $k$. Since $\sum_{\Pi^{\prime} \in \subseteq} \oplus Z \Pi / \Pi^{\prime}$ is $Z \Pi$-faithful, by (B), we have

$$
\mathfrak{a} \oplus \sum_{\Pi^{\prime} \in \subseteq} \oplus Z \Pi / \Pi^{\prime} \cong Z \Pi \oplus N
$$

for some $N$ with $N \approx \sum_{\Pi^{\prime} \in \mathscr{\Phi}} \oplus Z \Pi / \Pi^{\prime}$. Then, by (A) and (C), we can find $a_{\Pi^{\prime}}$ with $\mathfrak{a}_{\Pi^{\prime}} \sim Z \Pi / \Pi^{\prime}$ for all $\Pi^{\prime} \in \mathfrak{S}_{0}$ such that

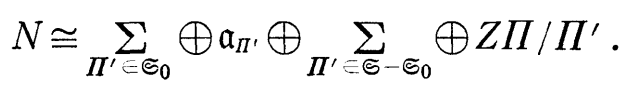

Hence we obtain that

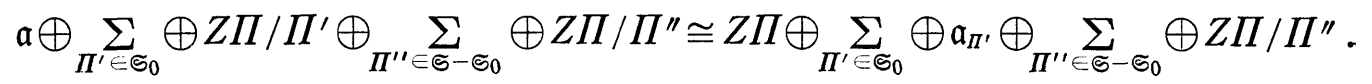

Thus $(*)_{0}$ is proved. Next suppose $(*)_{k}, k \geqq 0$. For each $\Pi^{\prime} \in \widetilde{\mathfrak{S}}_{k}, a_{\Pi^{\prime}}$ is considered as a $\Pi / \Pi^{\prime}$-module. We denote by $n^{\prime}$ the order of $\Pi / \Pi^{\prime}$. Then $\mathfrak{a}_{\Pi^{\prime}}^{\Phi} n^{\prime} \cong Z\left[\zeta_{n^{\prime}}\right]$. By (4.1) there exist a projective ideal $\mathfrak{c}_{\Pi^{\prime}}$ of $Z \Pi / \Pi^{\prime}$ with $\Omega_{\Pi / \Pi^{\prime}} c_{\Pi^{\prime}} \cong \Omega_{\Pi / \Pi^{\prime}}$, a permutation $\Pi / \Pi^{\prime}$-module $S_{\Pi^{\prime}}^{\prime}$ and a projective ideal $\mathfrak{a}_{\tilde{\Pi}^{\prime}}^{\left(I I^{\prime}\right)}$ of $Z \Pi / \tilde{\Pi}^{\prime}$ for any subgroup $\tilde{\Pi}^{\prime}$ of $\Pi$ such that $\tilde{\Pi}^{\prime} / \Pi^{\prime}$ is a cyclic group of prime order such that

$$
\mathfrak{a}_{\Pi^{\prime}} \bigoplus_{\widetilde{\Pi}^{\prime} \in \mathfrak{S}_{\Pi^{\prime}}} \oplus Z \Pi / \widetilde{\Pi}^{\prime} \oplus S_{\Pi^{\prime}}^{\prime} \cong \mathfrak{c}_{\Pi^{\prime}} \oplus{\widetilde{\widetilde{\Pi}^{\prime}} \in \sum_{\Pi^{\prime}}} \oplus \mathfrak{a}_{\widetilde{\Pi}^{\prime}}^{\left(\Pi^{\prime}\right)} \oplus S_{\Pi^{\prime}}^{\prime}
$$


where $\mathfrak{S}_{\Pi^{\prime}}$ denotes the set of all subgroups $\tilde{\Pi}^{\prime}$ of $\Pi$ such that $\Pi^{\prime} \subset \tilde{\Pi}^{\prime}$ and $\left[\tilde{\Pi}^{\prime}: \Pi^{\prime}\right]$ is a prime. However we have already proved the assertion in case $\Pi$ is cyclic. Therefore, for any $\Pi^{\prime} \in \widetilde{\Xi}_{k}$, there is a permutation $\Pi / \Pi^{\prime}$-module $S_{\Pi^{\prime}}^{\prime \prime}$ such that $c_{\Pi^{\prime}} \oplus S_{\Pi^{\prime}}^{\prime \prime} \cong Z \Pi / \Pi^{\prime} \oplus S_{\Pi^{\prime}}^{\prime \prime}$. Accordingly we have

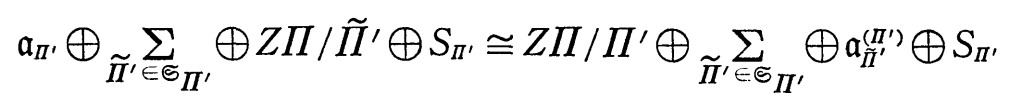

for some permutation $\Pi / \Pi^{\prime}$-module $S_{\Pi^{\prime}}$. From this and $\left({ }^{*}\right)_{k}$ it follows immediately that

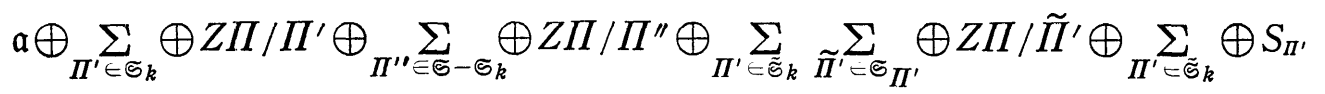

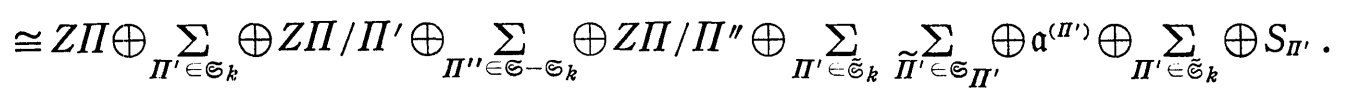

It is easily seen that any $\tilde{\Pi}^{\prime}$ of $\bigcup_{\Pi^{\prime} \in \tilde{\Phi}_{k}} \Im_{\Pi^{\prime}}$ contains some $\Pi^{\prime \prime}$ of $\mathfrak{S}_{k+1}$. According to (B), for fixed $\Pi^{\prime \prime} \in \Im_{k+1}$, we have

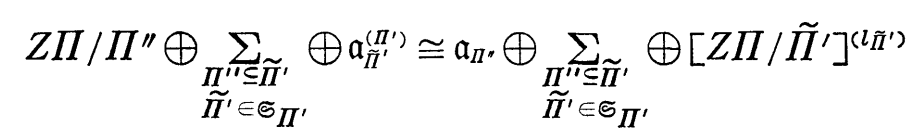

for a projective ideal $\mathfrak{a}_{\Pi^{\prime}}$ of $Z \Pi / \Pi^{\prime \prime}$ and $l_{\tilde{\Pi}^{\prime}} \geqq 0$. Hence from (**) we obtain

$$
\begin{aligned}
& \mathfrak{a} \oplus_{\Pi^{\prime \prime} \in \Phi_{k+1}} \oplus Z \Pi / \Pi^{\prime \prime} \oplus \sum_{\Pi^{\prime \prime \prime} \in \sum_{-}-\Im_{k+1}} \oplus Z \Pi / \Pi^{\prime \prime \prime} \oplus T
\end{aligned}
$$

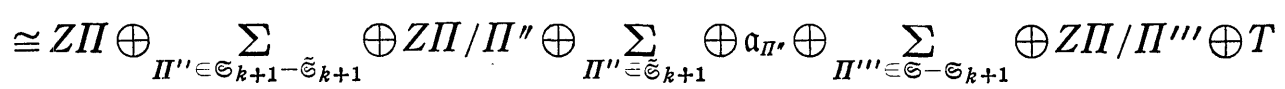

for some permutation $\Pi$-module $T$ such that $T \cong \sum_{\Pi^{\prime} \in \mathbb{S}} \oplus\left[Z \Pi / \Pi^{\prime}\right]^{\left(j_{\Pi^{\prime}}\right)}, j_{\Pi^{\prime}} \geqq 0$. Since $\mathfrak{a} \oplus \sum_{\Pi^{\prime} \in \Subset} \oplus Z \Pi / \Pi^{\prime} \sim Z \Pi \oplus \sum_{\Pi^{\prime} \in \Subset} \oplus Z \Pi / \Pi^{\prime}$, applying (D), we can omit $T$ from both sides, i. e.,

$$
\begin{aligned}
& \mathfrak{a} \oplus \sum_{\Pi^{\prime \prime} \in \mathfrak{S}_{k+1}} \oplus Z \Pi / \Pi^{\prime \prime} \oplus \sum_{\Pi^{\prime \prime \prime} \in \mathfrak{S}_{-\Im_{k+1}}} \oplus Z \Pi / \Pi^{\prime \prime \prime}
\end{aligned}
$$

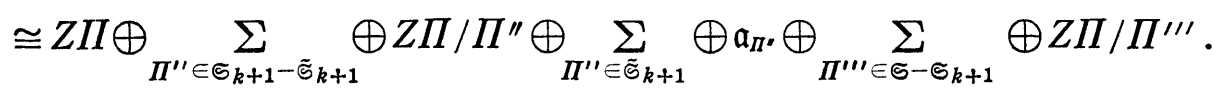

Thus we obtain $(*)_{k+1}$, which completes the proof.

COROLlaRY 4.3. Let $\Pi$ be a finite abelian group. Then

$$
\left|\gamma_{Z \Pi \oplus_{\Pi^{\prime} \in \mathscr{S}}^{\Sigma} \oplus \boldsymbol{Z} \Pi / \Pi^{\prime}}\right|=1 \text {, }
$$

where $\mathbb{S}$ denotes the set of all subgroups, $\Pi^{\prime}$, of $\Pi$ such that $\Pi / \Pi^{\prime}$ is cyclic.

$\S 5$. In $\S \S 5$ and 6 we will study non-projective quasi-permutation modules over finite cyclic groups.

Let $\Pi$ be a cyclic group of order $n$. Let $m \mid n$ and let $\Phi_{m}(T)$ be the $m$-th 
cyclotomic polynomial. We put $\Psi_{m}(T)=\left(T^{n}-1\right) / \Phi_{m}(T)$.

We begin with

LEMMA 5.1. Let $\Pi$ be a cyclic group of order $n$. Let $M$ be a $\Pi$-module. Then, for any $m \mid n,\left(M^{\Phi_{m}}\right) * \cong \Psi_{m} M^{*}$.

Proof. Consider the exact sequence: $0 \rightarrow M^{\Phi_{m}} \rightarrow M \rightarrow M / M^{\Phi_{m}} \rightarrow 0$. By dualizing this sequence we get an exact sequence

$$
0 \longrightarrow\left(M / M^{\Phi_{m}}\right)^{*} \longrightarrow M^{*} \longrightarrow\left(M^{\Phi_{m}}\right)^{*} \longrightarrow 0 \text {. }
$$

Then we have $\Psi_{m}\left(M / M^{\Phi_{m}}\right)^{*}=0$ and therefore there is an epimorphism: $\left(M^{\Phi_{m}}\right)^{*} \rightarrow \Psi_{m} M^{*}$. However $\operatorname{rank}_{Z}\left(M^{\Phi_{m}}\right)^{*}=\operatorname{rank}_{Z} \Psi_{m} M^{*}$. Hence $\Psi_{m} M^{*} \cong\left(M^{\Phi_{m}}\right)^{*}$.

Proposition 5.2. Let $\Pi$ be a cyclic group of order $n$. Then the following conditions are equivalent:

(1) For any $m \mid n$, all prime divisors of $n$ in $Z\left[\zeta_{m}\right]$ are principal.

(2) For any exact sequence $0 \rightarrow M^{\prime} \rightarrow M \rightarrow M^{\prime \prime} \rightarrow 0$ of $\Pi$-modules and any $m \mid n, M^{\Phi_{m}} \cong M^{\Phi_{m}} \oplus M^{\prime \prime \Phi_{m}}$.

(3) For any $\Pi$-module $M$ and any $m \mid n, M^{\Phi_{m}} \cong \Psi_{m} M$.

(4) For any $\Pi$-module $M$ and any $m \mid n,\left(M^{*}\right)^{\Phi_{m}} \cong\left(M^{\Phi_{m}}\right)^{*}$.

If $n$ is a prime power or if the class number of $Q\left(\zeta_{n}\right)$ is 1 , then the above conditions are satisfied.

Proof. The implications $(1) \Rightarrow(2)$ and $(1) \Rightarrow(3)$ were proved by Swan ([12], p. 108), and the implication $(3) \Leftrightarrow(4)$ follows directly from (5.1). Let $m \mid n(n \neq 1)$ and let $\mathfrak{p}$ be a prime divisor of $n$ in $Z\left[\zeta_{m}\right]$. Then, by a direct computation, we see that $\Psi_{m}\left(\zeta_{m}\right) \in \mathfrak{p}$ and therefore there is a divisor $d \neq m$ of $n$ such that $\Phi_{d}\left(\zeta_{m}\right) \in \mathfrak{p}$. Now put $A=Z\left[\zeta_{m}\right] / \mathfrak{p}$. Since $A$ can be considered as a $Z\left[\zeta_{d}\right]$-module, we can construct an exact sequence : $0 \rightarrow \mathfrak{q} \rightarrow Z\left[\zeta_{d}\right] \rightarrow A \rightarrow 0$ where $q$ is an ideal of $Z\left[\zeta_{d}\right]$. Forming the pullback as $Z \Pi$-modules, we get the following commutative diagram with exact rows and columns:

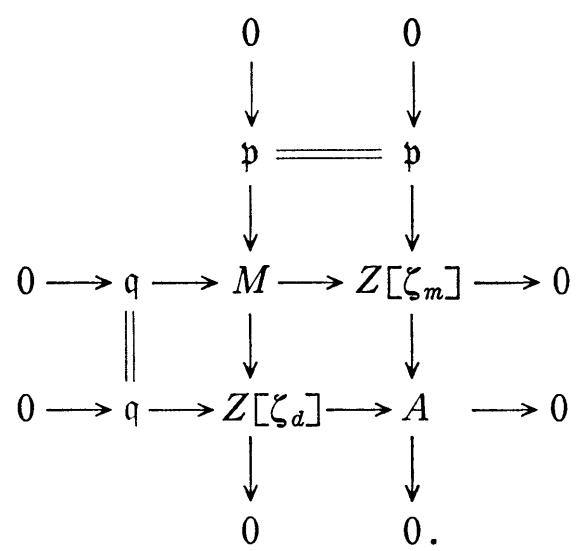

Then $M^{\Phi_{d}} \cong \mathfrak{q}$ and $M^{\Phi_{m}} \cong \mathfrak{p}$. Suppose that the condition (2) is satisfied. Because of the exactness of the second row, we have $M^{\Phi_{m}} \cong Z\left[\zeta_{m}\right]$, hence $\mathfrak{p} \cong$ $Z\left[\zeta_{m}\right]$, which implies (1). On the other hand, suppose that (4) is satisfied. 
By dualizing the second row, we have an exact sequence $0 \rightarrow Z\left[\zeta_{m}\right] \rightarrow M^{*} \rightarrow$ $\mathfrak{q}^{-1} \rightarrow 0$. Hence $\left(M^{*}\right)^{\Phi_{m}} \cong Z\left[\zeta_{m}\right]$. Since $\left(M^{*}\right)^{\Phi_{m}} \cong\left(M^{\Phi_{m}}\right)^{*}, \mathfrak{p} \cong Z\left[\zeta_{m}\right]$, which implies also (1). The second part of the proposition is obvious. Thus the proof of the proposition is completed.

Recently T. Sumioka ([10]) proved the equivalence of (1) and (2) in (5.2) in a little more general form.

We should remark that the smallest integer $n$ which does not satisfy the conditions in $(5.2)$ is $39=3 \cdot 13$. In fact, $\mathfrak{p}=\left(\zeta_{39}-3,13\right)$ is a non-principal prime ideal of $Z\left[\zeta_{39}\right]$ which divides 39 .

LEMMA 5.3 ([13]). Let $\Pi$ be a cyclic group of order $n$ and let $0 \rightarrow M \rightarrow N$ $\rightarrow S \rightarrow 0$ be an exact sequence where $M, N$ are $\Pi$-modules and $S$ is a permutation $\Pi$-module. Then $N^{\Phi_{n}} \cong M^{\Phi_{n}} \oplus S^{\Phi_{n}} \cong M^{\Phi_{n}} \oplus Z\left[\zeta_{n}\right]^{(t)}$ for some $t \geqq 0$. Especially, if $M$ is a quasi-permutation $\Pi$-module, then $M^{\Phi_{n}}$ is a free $Z\left[\zeta_{n}\right]$-module.

Proposition 5.4. Let $\Pi$ be a cyclic group of order $n$ and let $M$ be $a \Pi$ module. Then we have $M-{ }_{(r)} M^{\Phi_{n}} \oplus M / M^{\Phi_{n}}$. Especially $M$ is a quasi-permutation $\Pi$-module if and only if $M^{\Phi_{n}}$ is a free $Z\left[\zeta_{n}\right]$-module and $M / M^{\Phi_{n}}$ is a quasi-permutation $\Pi$-module.

PROOF. The second part of the proposition follows directly from the first part and (5.3). Hence we have only to prove the first part. First suppose that $M^{\Phi_{n}}$ is $Z\left[\zeta_{n}\right]$-free. Let $t$ be the rank of $M^{\Phi_{n}}$. Then we have an exact sequence

$$
0 \longrightarrow M^{\Phi_{n}} \longrightarrow Z \Pi^{(t)} \longrightarrow\left(Z \Pi /\left(\Psi_{n}(\sigma)\right)\right)^{(t)} \longrightarrow 0
$$

where $\sigma$ denotes a generator of $\Pi$. Put $F=Z \Pi^{(t)}$ and $L=\left(Z \Pi /\left(\Psi_{n}(\sigma)\right)\right)^{(t)}$. Using the same method as in the proof of (2.3) we can show that $L$ is a quasi-permutation $\Pi$-module. Since $\hat{H}^{0}\left(\Pi^{\prime}, Z\left[\zeta_{n}\right]\right)=0$ for any subgroup $\Pi^{\prime}$ of $\Pi$, we have $\hat{H}^{1}\left(\Pi^{\prime}, L\right)=0$ for any subgroup $\Pi^{\prime}$ of $\Pi$. Hence, by (1.6), there exist permutation $\Pi$-modules $S, S^{\prime}$ such that $L \oplus S^{\prime} \cong S$. Forming the pushout of $M^{\Phi_{n}} \rightarrow F$ and $M^{\Phi_{n}} \rightarrow M$, we get the following commutative diagram with exact rows and columns:

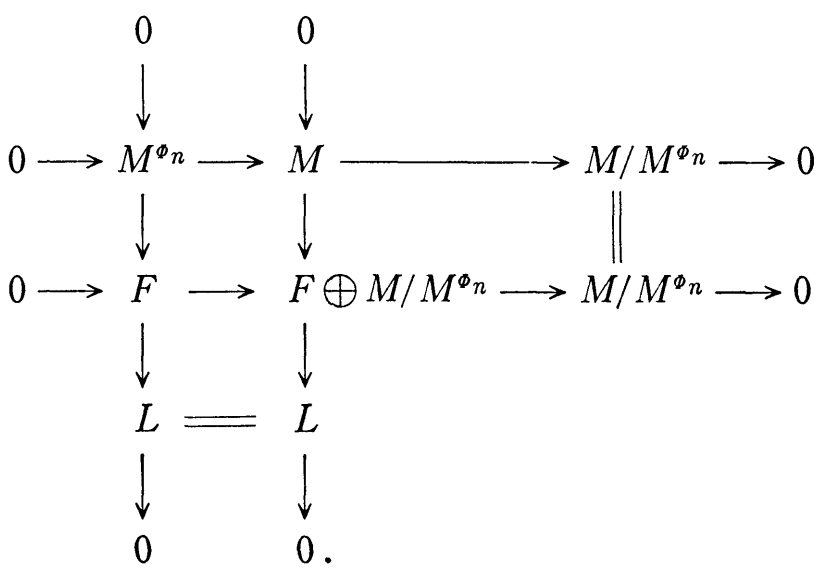


From the second column, we get an exact sequence

$$
0 \longrightarrow M \longrightarrow S^{\prime} \oplus F \oplus M / M^{\omega_{n}} \longrightarrow S \longrightarrow 0 \text {. }
$$

According to (1.3), we have $M-{ }_{(r)} S^{\prime} \oplus F \oplus M / M^{\Phi_{n}}-\overline{r r}-M / M^{\Phi_{n}}$, and therefore $M-(r)-M / M^{\Phi_{n}}-{ }_{(r)} M^{\Phi_{n}} \oplus M / M^{\Phi_{n}}$. In the general case we put $M_{0}=M \oplus\left(M^{\Phi_{n}}\right)^{*}$. Then $M_{0} / M_{0}^{\Phi_{n}} \cong M / M^{\Phi_{n}}$ and $M_{0}^{\Phi_{n}} \cong M^{\Phi_{n}} \oplus\left(M^{\Phi_{n}}\right)^{*}$. Since $M^{\Phi_{n}} \oplus\left(M^{\Phi_{n}}\right)^{*}$ is $Z\left[\zeta_{n}\right]$-free, $M_{0}^{\Phi_{n}}$ is $Z\left[\zeta_{n}\right]$-free. Therefore we have $M \oplus\left(M^{\Phi_{m}}\right)^{*} \cong M_{0}-{ }_{(r)} M_{0} / M_{0}^{\Phi_{n}}$ $\cong M / M^{\Phi_{n}}$, and hence $M \oplus\left(M^{\Phi_{n}}\right)^{*} \oplus M^{\Phi_{n}}-{ }_{(r)} M / M^{\Phi_{n}} \oplus M^{\Phi_{n}}$. Because $M^{\Phi_{n}} \oplus$ $\left(M^{\Phi_{n}}\right)^{*}$ is $Z\left[\zeta_{n}\right]$-free, this shows that $M-\frac{(r)}{(r)} M / M^{\Phi_{n}} \oplus M^{\Phi_{n}}$. Thus the proof of the proposition is completed.

THEOREM 5.5. Let $\Pi$ be a cyclic p-group of order $p^{l}$.

(0) Let $M$ be a $\Pi$-module. Then $M-(r)-\sum_{m=1}^{l} \oplus M^{\Phi_{p} m}$.

(1) A II-module $M$ is a quasi-permutation $\Pi$-module if and only if, for any $1 \leqq m \leqq l, M^{\Phi_{p} m}$ is $Z\left[\zeta_{p m}\right]$-free.

(2) If $M$ is a quasi-permutation $I I$-module, then the dual module $M^{*}$ is also a quasi-permutation $\Pi$-module.

(3) Let $0 \rightarrow M^{\prime} \rightarrow M \rightarrow M^{\prime \prime} \rightarrow 0$ be an exact sequence of $\Pi$-modules. If two of $M^{\prime}, M$ and $M^{\prime \prime}$ are quasi-permutation $\Pi$-modules, then the rest of them is also a quasi-permutation $\Pi$-module.

PROOF. We will prove (0) by induction on $l$. For $l=0$ this is obvious. Suppose that $l \geqq 1$. Now, by (5.4), we have $M-{ }_{(r)} M^{\Phi_{p} l} \oplus M / M^{\Phi_{p} l}$. Let $\Pi_{1}$ be the subgroup of $\Pi$ of order $p$. Then $M / M^{\phi_{p} l}$ can be regarded as a $\Pi / \Pi_{1^{-}}$ module. Therefore, by induction, $M / M^{\Phi_{p} l}-{ }^{(r)} \sum_{m=1}^{l-1} \oplus\left(M / M^{\Phi_{p} l}\right)^{\Phi_{p} m}$. However, by (5.2), $M^{\Phi_{p} m} \cong\left(M / M^{\Phi_{p} l}\right)^{\Phi_{p} m}$ for any $0 \leqq m \leqq l-1$, and so $M / M^{\Phi_{p} l}-\frac{}{(r)} \sum_{m=1}^{l-1} \oplus M^{\Phi_{p} m}$. Thus we get $M-\frac{}{(r)} \sum_{m=1}^{l} \oplus M^{\varphi_{p}}{ }^{m}$.

The assertion (1) follows directly from (0) and (2.6) (or (5.4)), and both (2) and (3) are immediate consequences of (1) and (5.2).

COROLLARY 5.6. Let $\Pi$ be a cyclic p-group and let $\Omega_{\Pi}$ be the maximal order in $Q \Pi$ which contains $Z \Pi$. Then the abelian semigroup $T(\Pi)$ is a group isomorphic to $C\left(\Omega_{\Pi}\right)$.

More generally, for a cyclic group $\Pi$ of order $n$, we consider the following statements :

(1) A $\Pi$-module $M$ is a quasi-permutation $\Pi$-module if and only if, for any $m \mid n, M^{\Phi_{m}}$ is $Z\left[\zeta_{m}\right]$-free.

(2) If $M$ is a quasi-permutation $\Pi$-module, then $M^{*}$ is also a quasipermutation $\Pi$-module.

(3) Let $0 \rightarrow M^{\prime} \rightarrow M \rightarrow M^{\prime \prime} \rightarrow 0$ be an exact sequence of $\Pi$-modules. If two of $M^{\prime}, M$ and $M^{\prime \prime}$ are quasi-permutation $\Pi$-modules, then the rest of them is a quasi-permutation $\Pi$-module. 
We conjecture that, for any cyclic group of order $n$ which satisfies the conditions in (5.2), all of (1), (2) and (3) are true. However we did not succeed in proving this in the general case.

REMARK 5.7. Let $\Pi$ be a cyclic group of order $n$ which satisfies the conditions in (5.2). If (1) is true for $\Pi$, then both (2) and (3) are true for $\Pi$.

REMARK 5.8. Let $\Pi$ be a cyclic group of order $n$ which does not satisfy the conditions in (5.2). Then both (2) and (3) are false for $\Pi$.

REMARK 5.9. Let $p, q$ be primes such that $q \mid p-1$ and suppose that there is a non-principal prime divisor $q$ of $p$ in $Z\left[\zeta_{q}\right]$. Let $\Pi$ be a cyclic group of order $p q$. Then (1) is false for $\Pi$ (cf. [12]). The smallest pair of primes satisfying the assumption is $\{47,23\}$.

From these remarks it seems fairly difficult to generalize (5.5), (0) and (1) to any finite cyclic group.

Proposition 5.10. Let $\Pi=[\sigma]$ be a cyclic group of order $n$ and let $p^{l} \mid n$ where $p$ is a prime. Let $0 \rightarrow N \rightarrow M \rightarrow Z\left[\zeta_{p l}\right]^{(t)} \rightarrow 0$ be an exact sequence of $\Pi$ modules where $N$ has no non-zero element invariant under $\sigma^{p l-1}$ (when $l \geqq 1$ ) and $t$ is a non-negative integer. Let $K / k$ be a Galois extension with group $\Pi$. Then $K(M)$ can be identified with $K\left(N \oplus Z\left[\zeta_{p l}\right]^{(t)}\right)$.

Proof. It suffices to prove this in the case of $t=1$ and $l \geqq 1$. Let $n=p^{l} d$, $q=p^{l}-p^{l-1}$ and $r=p^{l-1}$. We can identify $K(M)$ with $K(N)\left(X_{1}, X_{2}, \cdots, X_{q}\right)$ with the action of $\Pi$ such that $\sigma\left(X_{i}\right)=X_{i+1}, 1 \leqq i \leqq q-1$ and $\sigma\left(X_{q}\right)=\sigma^{q}\left(X_{1}\right)$ $=\frac{a}{X_{1} X_{r+1} X_{2 r+1} \cdots X_{(p-2) r+1}}$ for some $a \in N$. Then $\sigma^{r}\left(\sigma^{q}\left(X_{1}\right)\right)=\sigma^{p l}\left(X_{1}\right)=$ $\frac{\sigma^{r} a}{a} \cdot X_{1}$. Since $\sigma^{n}\left(X_{1}\right)=\sigma^{d p^{l}}\left(X_{1}\right)=X_{1}$, we have

$$
\frac{\sigma^{r} a}{a} \cdot \sigma^{p^{l}}\left(\frac{\sigma^{r} a}{a}\right) \cdot \sigma^{2 p^{l}}\left(\frac{\sigma^{r} a}{a}\right) \cdots \cdots \cdot \sigma^{(d-1) p l}\left(\frac{\sigma^{r} a}{a}\right)=1 .
$$

Hence $a \cdot \sigma^{p l} a \cdot \sigma^{2 p l} a \cdots \sigma^{(d-1) p^{l}} a$ is an element of $N$ invariant under $\sigma^{r}$. By the assumption we have

$$
a \cdot \sigma^{p^{l}} a \cdot \sigma^{2 p^{l}} a \cdots \sigma^{(d-1) p^{l}} a=1 .
$$

If we put $K^{\prime}=K(N)^{\left[\sigma p l_{]}\right.}$, then $N_{K(N) / K^{\prime}}(a)=1$. By the Hilbert's theorem 90, there is $b \in K(N)$ such that $a=b / \sigma^{p l} b$. Further put $c=\sigma^{r} b / b$ and $Z_{i}=\sigma^{i-1} c \cdot X_{i}$, $1 \leqq i \leqq q$. Then $\sigma^{i-1} c \in K(N)$ and so $K(M)=K(N)\left(Z_{1}, Z_{2}, \cdots, Z_{q}\right)$. We easily see that $c \cdot \sigma^{r} c \cdot \sigma^{2 r} c \cdots \sigma^{(p-1) r} c=a^{-1}$ and so we get

$$
\sigma\left(Z_{q}\right)=\frac{1}{Z_{1} Z_{r+1} Z_{2 r+1} \cdots Z_{(p-2) r+1}} .
$$

Therefore the group generated by $Z_{1}, Z_{2}, \cdots, Z_{q}$ is isomorphic to $Z\left[\zeta_{p l}\right]$. Thus $K(M)$ can be identified with $K\left(N \oplus Z\left[\zeta_{p l}\right]\right)$.

COROLlARY 5.11. Let $\Pi=[\sigma]$ be a cyclic group of order $n$ and let $p^{l} \mid n$ where $p$ is a prime. Let $0 \rightarrow N \rightarrow M \rightarrow L \rightarrow 0$ be an exact sequence of $\Pi$-modules 
where $N$ has no non-zero element invariant under $\sigma^{p l-1}$ (when $l \geqq 1$ ) and $L$ is $a$ $Z\left[\zeta_{p l}\right]$-module. Then $M-(r)-N \oplus L$.

PROOF. We have an exact sequence

$$
0 \longrightarrow N \longrightarrow M \oplus L^{*} \longrightarrow L \oplus L^{*} \longrightarrow 0 \text {. }
$$

Since $L \oplus L^{*}$ is $Z\left[\zeta_{p l}\right]$-free, this sequence satisfies the assumption in (5.10) and hence $M \oplus L^{*} \frac{{ }_{(r)}}{(r)} \oplus L \oplus L^{*}$. Therefore $M \oplus L^{*} \oplus L{ }_{(r)} N \oplus L \oplus L^{*} \oplus L$. Since $L \oplus L^{*} \underset{(r)}{-} 0$, this shows that $M-{ }_{(r)} N \oplus L$.

Proposition 5.12. Let $\Pi$ be a cyclic group of order $p^{l} q$ where $p, q$ are distinct primes and $l$ is a positive integer. Let $M$ be a $\Pi$-module. Let $M_{1}=M^{q_{1}}, M_{2}$ $=M_{1}^{\Phi_{p}}, \cdots, M_{l}=M_{l-1}^{{ }^{\Phi_{p}}{ }^{l-1}}$ and $M_{1}^{\prime}=\Phi_{p l_{q}} M^{\Phi p l_{q}{ }^{\phi} p l-l_{q} \cdots \Phi_{q}}, M_{2}^{\prime}=\Phi_{p l-1 q} M_{1}^{\prime}, \cdots, M_{l}^{\prime}$ $=\Phi_{p q} M_{l-1}^{\prime}$. Then

$$
M-\overline{(r)}^{\Phi} M^{\Phi} l_{q} \oplus \sum_{i=1}^{l} \oplus M_{i}^{\prime \phi_{p l-i_{q}}} \oplus \sum_{j=1}^{l} \oplus \Psi_{p j} M_{j} .
$$

Especially $M$ is a quasi-permutation $\Pi$-module if and only if $M^{\oplus p l_{q}}$ is $Z\left[\zeta_{p l q}\right]$ free, each $M_{i}^{\prime \phi_{p l-i}}$ is $Z\left[\zeta_{p l-i_{q}}\right]$-free and each $\Psi_{p_{j}} M_{j}$ is $Z\left[\zeta_{p^{j}}\right]$-free.

PROOF. We consider the following exact sequences:

$$
\begin{aligned}
& 0 \longrightarrow M_{1} \longrightarrow M \longrightarrow \Psi_{1} M \longrightarrow 0 \\
& 0 \longrightarrow M_{2} \longrightarrow M_{1} \longrightarrow \Psi_{p} M_{1} \longrightarrow 0 \\
& 0 \longrightarrow M_{l} \longrightarrow M_{l-1} \longrightarrow \Psi_{p l-1} M_{l-1} \longrightarrow 0 \\
& 0 \longrightarrow M_{l}^{\Phi^{p l}} \longrightarrow M_{l} \longrightarrow \Psi_{p l} M_{l} \longrightarrow 0 \text {. }
\end{aligned}
$$

Here $\Psi_{1} M$ is expressible as a direct sum of the copies of the trivial $\Pi$ module $Z$ and, for each $1 \leqq j \leqq l, \Psi_{p^{j}} M_{j}$ can be regarded as a $Z\left[\zeta_{p l}\right]$-module. Let $\sigma$ be a generator of $\Pi$. Then $M_{j}$ has no non-zero element invariant under $\sigma^{p^{j-1}}$. Therefore, according to (5.11), $M-{ }_{(r)} M_{1}$ and, for each $1 \leqq j \leqq l$, $M_{j}-(r)-M_{j+1} \oplus \Psi_{p j} M_{j}$. Thus we get

$$
M-(r) M_{l}{ }^{r_{p} l} \oplus \sum_{j=1}^{l} \oplus \Psi_{p j} M_{j} .
$$

We easily see that $M_{l}^{\Psi_{p} l}=M^{\Phi_{p} q^{\Phi} p l-1} q^{\cdots} \Phi_{q}$. Put $M^{\prime}=M_{l}^{\Psi_{p} l}$. Consider the following exact sequences :

$$
\begin{aligned}
& 0 \longrightarrow M^{\prime \Phi} p l q \longrightarrow M^{\prime} \longrightarrow M_{1}^{\prime} \longrightarrow 0 \\
& 0 \longrightarrow M_{1}^{\prime \Phi} p l-1 q \longrightarrow M_{1}^{\prime} \longrightarrow M_{2}^{\prime} \longrightarrow 0
\end{aligned}
$$




$$
\begin{aligned}
& 0 \longrightarrow M_{l-1}^{\prime{ }_{p q}} \longrightarrow M_{l-1}^{\prime} \longrightarrow M_{l}^{\prime} \longrightarrow 0 \\
& 0 \longrightarrow M_{l}^{\prime \Phi_{q}} \longrightarrow M_{l}^{\prime} \longrightarrow 0 .
\end{aligned}
$$

Here $M^{\prime \Phi p l q}=M^{\Phi} p l q$. Then, by (5.4), we have $M^{\prime}-(r) M^{\Phi} p l_{q} \oplus M_{1}^{\prime}$. Let $\Pi_{i}^{\prime}$ be the subgroup of $\Pi$ of order $p^{i}$, for each $1 \leqq i \leqq l$. Then $M_{i}^{\prime}$ can be regarded as a $\Pi / \Pi_{i}^{\prime}$-module. By virtue of (5.4), we have $M_{i}^{\prime}-{ }^{(r)} M_{i}^{\prime \Phi}{ }^{l-i_{q}} \oplus M_{i+1}^{\prime}$. Hence we get

$$
M^{\prime}-{ }_{(r)} M^{\Phi} p l_{q} \oplus \sum_{i=1}^{l} \oplus M_{i}^{\prime \phi p l-i_{q}} .
$$

Consequently we get

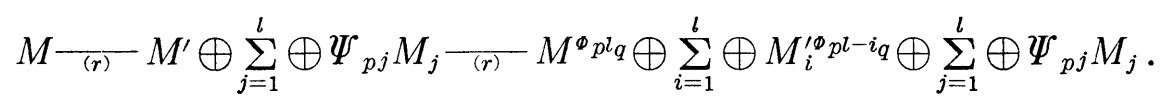

This completes the proof of the first part. The second part of the proposition follows immediately from the first part and (2.6).

LEMMA 5.13. Let $\Pi$ be a cyclic group of order $n$ and let $m_{1}, m_{2}$ be divisors of $n$ such that $m_{1}+m_{2}$ and $m_{2}+m_{1}$. Then $\operatorname{Ext}_{Z I}^{1}\left(M_{1}, M_{2}\right)=0$ for any $Z\left[\zeta_{m_{1}}\right]$ module $M_{1}$ and any $Z\left[\zeta_{m_{2}}\right]$-module $M_{2}$.

Proof. From the fact that $\left(\Phi_{m_{1}}(T), \Phi_{m_{2}}(T)\right)=Z[T]$ this follows immediately.

PROPOSITION 5.14. Let $\Pi$ be a cyclic group of order $p_{1} p_{2} p_{3}$ where $p_{1}, p_{2}, p_{3}$ are distinct primes. Let $M$ be a $\Pi$-module. Let $M^{\prime}=\Phi_{p_{1} p_{2} p_{3}} M^{\Phi_{1} p_{2} p_{3} \Phi_{p_{1}} p_{2} \Phi_{1} p_{3} \Phi_{p_{2}} p_{3}}$ and $M^{\prime \prime}=M^{\Phi_{1}}$. Then

$$
M-{ }_{(r)} M^{\Phi p_{1} p_{2} p_{3}} \oplus M^{\prime \Phi p_{1} p_{2}} \oplus M^{\prime \Phi p_{1} p_{3}} \oplus M^{\prime \Phi p_{2} p_{3}} \oplus \Psi_{p_{1}} M^{\prime \prime} \oplus \Psi_{p_{2}} M^{\prime \prime} \oplus \Psi_{p_{3}} M^{\prime \prime} .
$$

Especially $M$ is a quasi-permutation $\Pi$-module if and only if $M^{\Phi_{1} p_{2} p_{3}}$ is $Z\left[\zeta_{p_{1} p_{2} p_{3}}\right]$-free, each $M^{\prime \Phi_{i} p_{j}}$ is $Z\left[\zeta_{p_{i} p_{j}}\right]$-free and each $\Psi_{p_{i}} M^{\prime \prime}$ is $Z\left[\zeta_{p_{i}}\right]$-free.

PROOF. We have only to prove the first part. Consider the exact sequence $0 \rightarrow M^{\prime \prime} \rightarrow M \rightarrow \Psi_{1} M \rightarrow 0$. Then $\Psi_{1} M \cong Z^{(t)}$ for some $t \geqq 0$ and so $M-{ }_{(r)} M^{\prime \prime}$. Now put $\Phi=\Phi_{p_{1} p_{2} p_{3}} \cdot \Phi_{p_{1} p_{2}} \cdot \Phi_{p_{1} p_{3}} \cdot \Phi_{p_{2} p_{3}}$. Then $M^{\Phi}=M^{\prime \prime \Phi}$. Hence we have an exact sequence $0 \rightarrow M^{\Phi} \rightarrow M^{\prime \prime} \rightarrow \Phi M^{\prime \prime} \rightarrow 0$. Then $\Phi M^{\prime \prime}$ can be regarded as a $Z \Pi /\left(\Phi_{p_{1}}(\sigma) \Phi_{p_{2}}(\sigma) \Phi_{p_{3}}(\sigma)\right)$-module where $\sigma$ denotes a generator of $\Pi$. Therefore by (5.13) we can write $M^{\prime \prime}=M_{1} \oplus M_{2} \oplus M_{3}$ where each $M_{i}$ is a $Z\left[\zeta_{p_{i}}\right]$-module. Each $M_{i}$ is clearly isomorphic to $\Psi_{p_{i}} M^{\prime \prime}$. Applying (5.11) repeatedly, we get

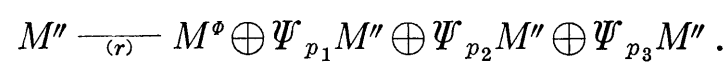

Next consider the exact sequence $0 \rightarrow M^{\Phi} p_{1} p_{2} p_{3} \rightarrow M^{\Phi} \rightarrow M^{\prime} \rightarrow 0$. According to (5.4), $\quad M^{\Phi}-(r) M^{\Phi_{1} p_{2} p_{3}} \oplus M^{\prime}$. Since $M^{\prime}$ can be regarded as a $Z \Pi /\left(\Phi_{p_{1} p_{2}}(\sigma) \Phi_{p_{1} p_{3}}(\sigma) \Phi_{p_{2} p_{3}}(\sigma)\right)$-module, again by (5.13) we can write $M^{\prime}=M_{12} \oplus$ $M_{13} \oplus M_{23}$ where each $M_{i j}$ is a $Z\left[\zeta_{p_{i} p_{j}}\right]$-module. It is easily seen that $M_{i j} \cong$ $M^{\prime \Phi} p_{i} p_{j}$. Hence we have

$$
M^{\Phi}-{ }_{(r)} M^{\Phi_{p_{1}} p_{2} p_{3}} \oplus M^{\prime \Phi p_{1} p_{2}} \oplus M^{\prime \Phi p_{1} p_{3}} \oplus M^{\prime \Phi p_{2} p_{3}} .
$$


Thus we get

$M-(r)-M^{\varphi_{p_{1} p_{2} p_{3}}} \oplus M^{\prime \phi_{p_{1} p_{2}}} \oplus M^{\prime \Phi_{1} p_{3}} \oplus M^{\prime \phi_{p_{2} p_{3}}} \oplus \Psi_{p_{1}} M^{\prime \prime} \oplus \Psi_{p_{2}} M^{\prime \prime} \oplus \Psi_{p_{3}} M^{\prime \prime}$.

COROllary 5.15. Let $\Pi$ be a cyclic group as in (5.12) or (5.14) and let $\Omega_{\Pi}$ be the maximal order in $Q \Pi$ containing $Z \Pi$. Then the abelian semigroup $T(\Pi)$ is a group isomorphic to $C\left(\Omega_{\Pi}\right)$.

§6. The following lemma is due to P. Samuel ([9]).

LEMma 6.1. Let $k$ be an infinite field and let $K_{1}, K_{2}$ be extensions of $k$ finitely generated over $k$. Suppose that there exist elements $x_{1}, x_{2}, \cdots, x_{n}$ which are algebraically independent over $K_{1}$ and $K_{2}$ such that $K_{1}\left(x_{1}, x_{2}, \cdots, x_{n}\right)=$ $K_{2}\left(x_{1}, x_{2}, \cdots, x_{n}\right)$. Then $K_{1}$ is k-isomorphic to $K_{2}$.

LEMMA 6.2. Let $\Pi$ be a cyclic p-group of order $p^{l}$ and let $K / k$ be a Galois extension with group $\Pi$. In case of $p \neq 2$, suppose that $k$ is an infinite field. Then $K\left(Z\left[\zeta_{p l}\right]\right)^{\Pi}$ is rational over $k$.

PROOF. For $l=1$ this has been proved in [2], (1.13). Hence we may suppose that $l \geqq 2$. Let $q=p^{l-1}$ and let $\sigma$ be a generator of $\Pi$.

(i) Case of $p=2$. Take $b \in K$ such that $\sigma^{q} b \neq b$ and put $a=b / \sigma^{q} b$. Then $a \cdot \sigma^{q} a=1$ and $\sigma^{q} a \neq a$. Now $K\left(Z\left[\zeta_{2 l}\right]\right)$ is expressed as the rational function field $K\left(X_{1}, X_{2}, \cdots, X_{q}\right)$ with the action of $\Pi$ such that $\sigma\left(X_{i}\right)=X_{i+1}, 1 \leqq i \leqq q-1$ and $\sigma\left(X_{q}\right)=1 / X_{1}$. If we put $Y_{1}=\frac{X_{1}+a}{X_{1}+\sigma^{q} a}$ and $Y_{i+1}=\sigma\left(Y_{i}\right), 1 \leqq i \leqq q-1$, then $K\left(Z\left[\zeta_{2} l\right]\right)=K\left(Y_{1}, Y_{2}, \cdots, Y_{q}\right)$ and $\sigma\left(Y_{q}\right)=\frac{\sigma^{q} a}{a} Y_{1}$, and therefore $\Pi$ acts semilinearly on $\sum_{i=1}^{q} K Y_{i}$. Thus, by $(1.1), K\left(Z\left[\zeta_{2} l\right]\right)^{I}$ is rational over $k$.

(ii) Case of $p \neq 2$. Suppose that $k$ is an infinite field. By the definition $K(Z \Pi)$ is the rational function field $K\left(X_{1}, X_{2}, \cdots, X_{p l}\right)$ with the action of $\Pi$ such that $\sigma\left(X_{i}\right)=X_{i+1}, 1 \leqq i \leqq p^{l}-1$ and $\sigma\left(X_{p l}\right)=X_{1}$. Then $K\left(Z\left[\zeta_{p l}\right]\right)$ can be identified with $K\left(X_{q+1}^{-1} X_{1}, X_{q+2}^{-1} X_{2}, \cdots, X_{p l}^{-1} X_{q(p-1)}\right)$ because $Z\left[\zeta_{p l}\right]=\left(\sigma^{q}-1\right)$, and we have $K(Z \Pi)=K\left(Z\left[\zeta_{p l}\right]\right)\left(X_{1}, X_{2}, \cdots, X_{q}\right)$. Let

$$
Y=\frac{X_{1}+X_{q+1}+X_{2 q+1}+\cdots+X_{(p-1) q+1}}{X_{1}} .
$$

Then $Y \in K\left(Z\left[\zeta_{p l}\right]\right)$. Further let $Z_{1}=X_{1}+X_{q+1}+X_{2 q+1}+\cdots+X_{(p-1) q+1}$ and $Z_{i+1}$ $=\sigma^{i} Z_{1}, 1 \leqq i \leqq q-1$. Then $\sigma^{q} Z_{1}=Z_{1}$ and $Z_{1}, Z_{2}, \cdots, Z_{q}$ are algebraically independent over $K\left(Z\left[\zeta_{p l}\right]\right)$ since $Z_{i}=\sigma^{i-1}(Y) \cdot X_{i}, 1 \leqq i \leqq q$. Now put $V=\sum_{j=1}^{p l} K X_{j}$ and $W=\sum_{i=1}^{q} K Z_{i}$. Then $W \leqq V$ and $\Pi$ acts semi-linearly on $V$ and $W$. By (1.1) there exist $U_{1}, U_{2}, \cdots, U_{q} \in K(W)$ which are invariant under $\Pi$ such that $K(W)=K\left(U_{1}, U_{2}, \cdots, U_{q}\right)$. Then we have

$$
K(Z M)^{\Pi}=\left[K\left(Z\left[\zeta_{p l}\right]\right)(W)\right]^{\Pi}=K\left(Z\left[\zeta_{p l}\right]\right)^{I}\left(U_{1}, U_{2}, \cdots, U_{q}\right) .
$$


On the other hand we have an exact sequence:

$$
0 \longrightarrow W \longrightarrow V \longrightarrow V / W \longrightarrow 0
$$

of $K$-vector spaces. Applying (1.1) to this we can find $U_{1}^{\prime}, U_{2}^{\prime}, \cdots, U_{q(p-1)}^{\prime}$ $\in K(V)$ such that $K(Z \Pi)^{\Pi}=K(V)^{\Pi}=K(W)^{\Pi}\left(U_{1}^{\prime}, U_{2}^{\prime}, \cdots, U_{q(p-1)}^{\prime}\right)$. Therefore we get

$$
\begin{aligned}
& K\left(Z\left[\zeta_{p l}\right]\right)^{\Pi}\left(U_{1}, U_{2}, \cdots, U_{q}\right)=K(Z \Pi)^{\Pi} \\
& \quad=k\left(U_{1}^{\prime}, U_{2}^{\prime}, \cdots, U_{q(p-1)}^{\prime}\right)\left(U_{1}, U_{2}, \cdots, U_{q}\right) .
\end{aligned}
$$

Here $U_{1}, U_{2}, \cdots, U_{q}$ are algebraically independent over $K\left(Z\left[\zeta_{p l}\right]\right)^{I I}$ and $k\left(U_{1}^{\prime}\right.$, $\left.U_{2}^{\prime}, \cdots, U_{q(p-1)}^{\prime}\right)$. Then, by virtue of $(6.1), K\left(Z\left[\zeta_{p l}\right]\right)^{\Pi}$ is $k$-isomorphic to the rational function field $k\left(U_{1}^{\prime}, U_{2}^{\prime}, \cdots, U_{q(p-1)}^{\prime}\right)$. This completes the proof of the lemma.

THEOREM 6.3. Let $\Pi$ be a cyclic p-group and let $K / k$ be a Galois extension with group II. In case of $p \neq 2$ suppose that $k$ is an infinite field. If $M$ is a quasi-permutation $\Pi$-module, then $K(M)^{I} / k$ is rational.

ProOF. Let $I$ be a cyclic group of order $p^{l}$. As in (5.12) we put $M_{0}=$ $M, M_{1}=M_{0}^{\Psi_{1}}, M_{2}=M_{1}^{\Psi_{p}}, \cdots, M_{l}=M_{l-1}^{q_{p} l-1}$. Then we have the following exact sequences:

$$
\begin{aligned}
& 0 \longrightarrow M_{1} \longrightarrow M_{0} \longrightarrow \Psi_{1} M_{0} \longrightarrow 0 \\
& 0 \longrightarrow M_{2} \longrightarrow M_{1} \longrightarrow \Psi_{p} M_{1} \longrightarrow 0 \\
& 0 \longrightarrow M_{l} \longrightarrow M_{l-1} \longrightarrow \Psi_{p l-1} M_{l-1} \longrightarrow 0 \\
& 0 \longrightarrow M_{l} \longrightarrow \Psi_{p l} M_{l} \longrightarrow 0 \text {. }
\end{aligned}
$$

By (5.2) we have $\Psi_{p^{i}} M_{i} \cong M^{\Phi_{p}}$ for each $0 \leqq i \leqq l$ and further, by (5.5), $M^{\Phi^{i}}$ is $Z\left[\zeta_{p^{i}}\right]$-free for each $0 \leqq i \leqq l$. Therefore, applying (5.10) to the above exact sequences repeatedly, we see that $K(M)$ is $k$-isomorphic to $K\left(M^{\Phi_{p} l} \oplus M^{\Phi_{p} l-1} \oplus\right.$ $\left.\cdots \oplus M^{\Phi_{1}}\right)$. Thus we can conclude by $(6.2)$ that $K(M)^{\Pi}$ is rational over $k$.

\section{References}

[1] S. B. Conlon, Monomial representations under integral similarity, J. Algebra, 13 (1969), 496-508.

[2] S. Endo and T. Miyata, Invariants of finite abelian groups, J. Math. Soc. Japan, 25 (1973), 7-26.

[3] H. Jacobinski, Genera and decompositions of lattices over orders, Acta Math., 121 (1968), 1-29.

[4] I. Reiner and S. Ullom, Class groups of integral group rings, Trans. Amer. Math. Soc., 170 (1972), 1-30. 
[5] D.S. Rim, On projective class groups, Trans. Amer. Math. Soc., 98 (1961), 459-467.

[6] A. V. Roiter, On integral representations belonging to a genus, Izv. Akad. Nauk SSSR, 30 (1966), 1315-1324.

[7] P. Roquette, Realisierung von Darstellungen endlicher nilpotenter Gruppen, Archiv. der Math., 9 (1958), 241-250.

[8] M. Rosen, Representations of twisted group rings, Thesis at Princeton Univ., 1963.

[9] P. Samuel, Some remarks on Lüroth's theorem, Mem. Univ. Kyoto, 27 (1953), 223-224.

[10] T. Sumioka, A note on the Grothendieck group of a finite group, to appear in Osaka J. Math.

[11] R. G. Swan, Induced representations and projective modules, Ann. of Math., 71 (1960), 552-578.

[12] R. G. Swan, The Grothendieck ring of a finite group, Topology, 2 (1963), 85-110.

[13] R.G. Swan, Invariant rational functions and a problem of Steenrod, Invent. Math., 7 (1969), 145-158.

[14] V. E. Voskresenskiǔ, Birational properties of linear algebraic groups, Izv. Akad. Nauk SSSR, 34 (1970), 3-19.

[15] V.E. Voskresenskir, On the question of the structure of the subfield of invariants of a cyclic group of automorphisms of the field $Q\left(x_{1}, \cdots, x_{n}\right)$, Izv. Akad. Nauk SSSR, 34 (1970), 366-375.

\section{Shizuo ENDo}

Department of Mathematics Tokyo Metropolitan University

Fukazawa-cho, Setagaya-ku,

Tokyo, Japan
Takehiko Miyata

Department of Mathematics

Osaka City University

Sugimoto-cho, Sumiyoshi-ku, Osaka, Japan 